\title{
Norois
}

Environnement, aménagement, société

$240 \mid 2016$

Aménagement foncier, risque côtier, évolution des fleuves

\section{Construction des lieux et rapports à la nature}

Cabanons et pêcheurs des lacs du Sud Manche

Places Building and Relationships with Nature. Lakeside Huts and Fishermen in the Sélune Valley, Normandy

\section{Marie-Anne Germaine, Matthieu Viry et Marie-Jo Menozzi}

\section{(2) OpenEdition}

\section{Édition électronique}

URL : https://journals.openedition.org/norois/5955

DOI : $10.4000 /$ norois. 5955

ISBN : 978-2-7535-5362-0

ISSN : $1760-8546$

Éditeur

Presses universitaires de Rennes

Édition imprimée

Date de publication : 30 novembre 2016

Pagination : $77-100$

ISBN : 978-2-7535-5356-9

ISSN : 0029-182X

\section{Référence électronique}




5 NOËB⺝S

\title{
Construction des lieux et rapports à la nature Cabanons et pêcheurs des lacs du Sud Manche ${ }^{1}$
}

\author{
Constructing Places and Relationships with Nature \\ Lakeside Huts and Fishing in the Sélune Valley, Normandy
}

Marie-Anne Germaine ${ }^{* a}$, Matthieu Viry ${ }^{\mathrm{b}}$ et Marie-Jo MenOzzI ${ }^{\mathrm{c}}$

\footnotetext{
*Auteur correspondant : Tel : (33)1 40-97-75-84 (marie-anne.germaine@u-paris10.fr)

a LAVUE - UMR 7218 CNRS, Université Paris Ouest Nanterre La Défense, 200 av. de la République, 92000

Nanterre, France.

b UMS 2414 RIATE CNRS-CGET, Université Paris-Diderot, 8 place Paul-Ricœur, 75013 PARIs, France. (matthieu. viry@gmail.com)

${ }^{c}$ Ethnozzi, 18 Painfaut, 56350 Saint-VIncent-sur-Oust, France. (marie-jo.menozzi@orange.fr)
}

Résumé : Alors que les collectivités territoriales du Sud Manche achèvent leur réflexion concernant le projet de reconversion de la vallée de la Sélune après l'arasement à venir des barrages hydroélectriques de Vezins et de la Roche-qui-Boit, cet article propose d'analyser les usages et représentations associés aux nombreux cabanons aménagés au bord de ces deux retenues d'eau. Développées dans les années 1960 en lien avec l'essor de la pêche de loisir, ces constructions constituent des lieux de villégiature populaire autour desquels s'est construit un certain rapport à la nature. Un inventaire exhaustif et la caractérisation de ces installations ont permis de construire une typologie révélant des points communs (auto-construction, liberté architecturale) derrière une hétérogénéité apparente et une diversité de statuts fonciers. Les cabanes apparaissent comme le lieu de pratiques contemplatives de la nature car elles offrent un refuge isolé mais elles constituent aussi l'espace d'une riche sociabilité liée à la pêche. Peu prises en compte par les pouvoirs publics qui les considèrent comme des points noirs paysagers, ces cabanes sont l'objet d'un fort attachement. L'analyse de leur histoire, des usages et représentations qui leur sont associées apportent des éléments utiles à la compréhension des enjeux actuels.

\begin{abstract}
This paper aims at discussing the history and uses of huts built on the banks of two lakes located in the Sélune Valley in southwest Normandy. While the lakes are soon to disappear, local authorities are developing a plan for the economic and social adaptation of the valley without these lakes. A number of huts were built in the 1960s, following the growing appeal for recreational fishing. An exhaustive inventory and characterization of these constructions was produced in the order to identify different types of huts. This typology reveals common features (self-building, architectural freedom) as well as a certain heterogeneity in terms of comfort and ownership status. These huts appears as places for contemplative practices in nature, but are also places of sociability related to fishing activities. The people we met are very attached to these huts, when institutionals actors regard them primarly as a blot on the landscape by institutional actors. Lastly, the analysis of the huts' history, uses and representations seems to provide useful elements for a better consideration of the valley's future.
\end{abstract}

Mots clés : cabane - pêche - plan d'eau - loisirs - représentation - nature

Keywords: hut - fishing - lake - leisure - representation - nature

1. Cet article a été rédigé en 2015 à partir d'enquêtes de terrain menées durant le printemps et l'été 2014, soit avant la suspension du projet d'effacement des barrages de la Sélune. Ce projet est aujourd’hui réduit à la vidange et à la gestion sédimentaire du barrage de Vezins (arrêté du 3 mars 2016 ). 


\section{INTRODUCTION}

Les constructions de type cabanons ont fait l'objet de nombreux travaux sociologiques et ethnologiques (Nicolas, 2008) en zone littorale comme sur la plage du Valais près de Saint-Brieuc ou la plage de Beauduc en Camargue (Claeys-Mekdade et Nicolas, 1999). Ce sont surtout les rapports homme/nature qui ont été étudiés dans ces travaux à travers l'analyse des représentations associées à ces constructions, aussi qualifiées d'habitats légers de loisir. Elles sont le plus souvent démontables et occupées temporairement durant la saison privilégiée pour la pratique d'activités récréatives de nature (Picon, 2001). Ces cabanes sont étudiées en lien avec un mode d'habiter dans la nature spécifique et associé aux loisirs populaires à l'instar du camping (Collectif, 2001). Peu de travaux se sont en revanche intéressés à caractériser la diversité des formes de ces constructions mais un ouvrage récent rassemblant des regards issus de plusieurs disciplines propose de faire de l'abri de loisir un objet d'étude en soi (Raveneau et Sirost, 2011). L'étude des conflits sociaux constitue l'autre pan important des recherches associées à ces constructions. Dénoncées comme des implantations informelles ou encore « sauvages » dans des sites naturels, ces cabanes sont remises en cause par l'État suscitant des tensions entre usagers et pouvoirs publics (Cadoret, 2006).

Bien qu'ils aient été moins étudiés, les bords de rivière et de plans d'eau constituent eux aussi des lieux privilégiés d'appropriation éphémère de la nature (Maugin et Perault, 2011). C'est le cas des berges des lacs de la Sélune situés à quelques kilomètres de la baie du Mont-Saint-Michel. Ces lacs sont bordés de constructions atypiques qui vont de l'abri de pêche à la résidence principale. Si l'histoire de ces constructions est devenue indissociable de celle des lacs, leur pérennité est remise en question depuis novembre 2009 lorsque l'État, motivé par la réglementation (Directive Cadre sur l'Eau) et la restauration de la continuité écologique pour les poissons migrateurs (les saumons en particulier), a annoncé l'effacement des deux barrages hydroélectriques qui retiennent ces lacs. Alors que l'État et les collectivités locales préparent la reconversion socioéconomique et paysagère de la vallée (Germaine et Lespez, 2014), le devenir de ces bâtiments demeure absent des réflexions. Dans le cadre d'un programme de recherche ${ }^{2}$ visant à suivre cette opération d'arasement d'ampleur inédite à l'échelle européenne, nous avons mené un travail d'enquête auprès des usagers de ces constructions. Ces cabanons apparaissent en effet comme un des témoignages les plus visibles de l'appropriation des lacs face au projet de suppression des barrages (et donc des lacs) qui fait l'objet d'une vive protestation locale. S'il permet d'éclairer les blocages contemporains, l'objet premier de cet article est de questionner les modalités, matérielles et idéelles, d'appropriation de l'espace des lacs par les usagers des cabanons (Frémont, 1976; Debarbieux, 1996; Ripoll et Veschambre, 2005. Lazzarotti, 2006). Ce rapport à l'espace est abordé à travers les représentations mais aussi les pratiques que les usagers mettent en œuvre dans l'espace (Tuan, 1974; Berque, 1994). Abordés à travers l'analyse des choix de construction et d'aménagement des lieux d'une part et des pratiques de l'espace d'autre part, les rapports pratiques à l'espace permettent de questionner les manières de faire avec l'espace et de faire l'espace et non pas seulement l'imaginaire (Stock, 2012). La démarche mobilisée emprunte ainsi au courant de la géographie culturelle tout en mettant en avant l'étude de la matérialité des formes habitées et des pratiques. Cette double entrée permet aussi de dépasser l'échelle individuelle pour aborder la dimension collective de ce rapport aux lieux (regard des institutions, valeurs sociales, normes...). Ce travail amène donc à discuter à la fois du rapport individuel que les usagers des cabanons entretiennent aux lacs et à la nature et du caractère exclusif de l'usage de ces lieux remis en cause par le projet écologique.

\section{Présentation du terrain d'Étude}

Au début du $\mathrm{xx}^{\mathrm{e}}$ siècle, deux barrages hydroélectriques ont été construits sur le cours aval de la Sélune - la Roche-qui-Boit (16 m de haut) et Vezins $(36 \mathrm{~m})$ - créant deux retenues d'eau longues d'environ 4 et $17 \mathrm{~km}$. Elles occupent la partie

2. Le programme «Restauration écologique de la rivière Sélune. Paysage, usages, représentations : quels changements après l'arasement des barrages de Vezins et de la Roche-qui-Boit? » est financé par l'Agence de l'eau Seine-Normandie (AESN) et piloté par M.-A. Germaine (université Paris Ouest Nanterre La Défense). Il s'agit d'un des quatre volets du programme de suivi scientifique de l'arasement des barrages de la Sélune piloté par J.-L. Baglinière (INRA). Consulter le site Internet : [http:// selune.hypotheses.org/]. 
moyenne de la Sélune avant que celle-ci ne rejoigne la Baie du Mont-Saint-Michel : il s'agit d'un tronçon étroit et boisé, encaissé d'environ 70 m par rapport aux plateaux agricoles environnants (figure 1 - planche VI). Ces gorges présentent des versants boisés et quelques escarpements rocheux qui renforcent le caractère secret et intime de ce paysage visible uniquement depuis quelques belvédères et les trois ponts qui traversent la rivière dans sa partie moyenne.

Les lacs de retenue sont surtout réputés pour la pratique de la pêche aux carnassiers (perche, brochet, sandre, silure) et poissons blancs (carpe, gardon et brème). Soutenues par une politique de promotion touristique portée par les élus locaux, d'autres activités récréatives ont été développées (Germaine et Lespez, 2014). La plus emblématique est aujourd'hui la base de loisirs de la Mazure : gérée par les collectivités locales, elle accueille surtout un public scolaire. Cependant, l'attractivité du site s'est surtout traduite par une valorisation privée des berges à travers l'installation de cabanons et pontons liés à la pêche. Ces constructions sont néanmoins peu connues du fait de leur discrétion dans le paysage.

\section{Méthodologie}

Ce travail a été réalisé à partir d'un inventaire des constructions en bordure des lacs intégré dans une base de données géo-référencées et d'une enquête menée auprès de pêcheurs des lacs ainsi que de propriétaires ou occupants des constructions.

L'inventaire a été réalisé à partir du cadastre, complété par l'analyse de photographies aériennes (1949 à 2010). Ces sources se sont révélées insuffisantes pour réaliser un inventaire complet, toutes les constructions n'étant pas déclarées et une partie d'entre elles étant invisibles sur les photos aériennes du fait de la densité de végétation. Le repérage s'est donc appuyé sur des sorties de terrain. Dans un périmètre de $200 \mathrm{~m}$ autour des lacs, les cabanes et pontons ont été localisés à l'aide d'un GPS, caractérisés selon une grille de description (tableau 1) puis reportés dans un Système d'Information Géographique.

Cet inventaire s'est accompagné de la conduite d'une enquête de type ethnosociologique menée durant le printemps et l'été $2014^{3}$ auprès des usa-

3. Soitavant la remise en cause du projet d'arasement intervenue suite à la venue en décembre 2014 de Madame la ministre de l'Environnement sur gers de cabanons (40) et des lacs (75 autres personnes). Nous avons mis à profit la présence sur le terrain à l'occasion de l'inventaire des cabanons pour effectuer des observations de visu et pour réaliser des entretiens avec les personnes croisées. La majorité d'entre eux $(80 \%)$ a été rencontrée sur le lac de Vezins, plus grand et plus fréquenté. Les trois quarts des usagers des cabanons et des pêcheurs interviewés ont été rencontrés de manière inopinée sur le terrain. Si la plupart d'entre eux a accepté de répondre à nos questions, la situation ne se prêtait qu'à une enquête rapide, à partir d'un guide composé de quelques questions. Du fait du caractère imprévu des rencontres et de la méfiance initiale des personnes rencontrées, il a souvent été difficile d'obtenir des caractéristiques précises sur leur statut (tableau 2). La plupart des usagers rencontrés sur la Sélune sont des ouvriers, employés, artisans ou commerçants. Ces enquêtes ont été complétées par la réalisation d'une douzaine d'entretiens semi-directifs plus approfondis (systématiquement enregistrés) auprès d'autres usagers de cabanons et pêcheurs avec lesquels nous avons pu fixer un rendez-vous.

La grille d'entretien utilisée avec les pêcheurs visait à caractériser leur fréquentation des lacs et de la rivière (usages, temporalités, activité individuelle ou collective) ainsi que leur perception de ces derniers (description, lieux préférés). Les projets concernant le devenir des barrages étaient ensuite abordés. La grille mobilisée auprès des usagers des cabanons incluait les mêmes thématiques mais aussi des questions visant à caractériser l'histoire et l'usage du cabanon. Le caractère temporaire de l'occupation des constructions a constitué une réelle difficulté pour la conduite de l'enquête : certaines sessions de terrain se sont révélées très infructueuses tandis que d'autres n'étaient pas assez longues pour rencontrer toutes les personnes présentes simultanément. Les discours collectés ont été analysés de façon d'une part à renseigner les pratiques, individuelles et collectives de l'espace, et d'autre part comprendre les représentations qui sous-tendent l'appropriation cognitive et affective des lacs.

Les représentants des associations locales ont été rencontrés. Alors qu'aucun des présidents des trois AAPPMA présentes le long de la Sélune n'est cabanonier, les membres du bureau de l'associa-

la Sélune. Les enquêtes ont par ailleurs souvent été menées en binôme associant une socio-anthropologue et un(e) géographe. 


\begin{tabular}{|c|c|c|}
\hline \multirow{4}{*}{ 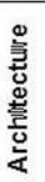 } & Matériaux de construction & Bois, tôle, béton, etc. \\
\hline & Surface & $m^{2}$ \\
\hline & État général du bâtiment & Délabré/mauvais / moyen /bon \\
\hline & Aménagement d'un jardin & Oui/Non \\
\hline \multirow{2}{*}{ 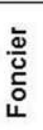 } & Présence du bâtiment sur le cadastre & Oui/Non \\
\hline & Propriétaire de la parcelle & EDF/privé propriétaire cabanon/autre privé \\
\hline \multirow{2}{*}{ 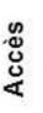 } & Type d'accès au bâtiment & Public/privé - chemin piéton, etc. \\
\hline & Signes de privatisation & Clôture, portail, panneaux, etc. \\
\hline \multirow{3}{*}{ 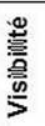 } & Visibilité du bâtiment depuis l'espace public & Oui / Non / Difficile \\
\hline & Visibilité du lac depuis le bâtiment & Oui / Non / Difficile \\
\hline & Visibilité de la rivière depuis le bâtiment & Oui / Non / Difficile \\
\hline \multirow{6}{*}{ 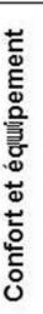 } & Présence d'un balcon / d'une terrasse / véranda & Oui (type) / Non \\
\hline & Présence d'un autre bâtiment sur la même parcelle & Abri de jardin, garage, etc. \\
\hline & Présence d'un ponton en lien & Oui (identifiant) / Non \\
\hline & Présence d'une embarcation & Oui/Non \\
\hline & Raccord aux réseaux & Électricité / Eau/Téléphone \\
\hline & Équipements divers & Antenne TV, ventilation, etc. \\
\hline \multirow{3}{*}{$\begin{array}{l}\stackrel{\infty}{\infty} \\
\stackrel{2}{2}\end{array}$} & \multicolumn{2}{|c|}{ Date de construction } \\
\hline & \multicolumn{2}{|c|}{ Nom donné au bâtiment / au site } \\
\hline & \multicolumn{2}{|c|}{ Coordonnées des occupants (et coordonnées du propriétaire de la parcelle le cas échéant) } \\
\hline
\end{tabular}

Tableau 1 : Critères renseignés dans la grille de relevés characterize huts

tion des Amis du barrage, qui milite contre le projet d'arasement, indiquent en revanche fréquenter régulièrement les cabanes même s'ils n'en possèdent pas tous eux-mêmes. En parallèle, des entretiens semi-directifs ont été menés auprès d'acteurs institutionnels, politiques et économiques : ces derniers ne traitaient pas spécifiquement de la question des cabanons mais la question a été abordée avec les maires des communes concernées, les services de l'État et des représentants d'EDF.

L'intérêt des acteurs institutionnels et des élus visà-vis des cabanons a également été abordé à travers un travail d'observation participante (Germaine et Lespez, 2014) et des entretiens réalisés auprès des élus locaux. Au titre de notre collaboration entre 2011/2013 avec le bureau d'études ETHEIS que la DDTM de la Manche avait chargé de réaliser le Schéma de développement durable de la vallée de la Sélune, nous avons participé activement à l'organisation d'ateliers réunissant divers acteurs locaux en vue d'établir un diagnostic du territoire. Nous avons également assisté (de manière plus passive cette fois) aux réunions du Comité de pilotage de la nouvelle étude socio-économique prescrit depuis 2014 par le Syndicat Mixte du Pays de la Baie ainsi qu'aux rares réunions d'information organisées par les services de l'État ou aux plus nombreuses réunions publique proposées par les Amis du barrage.

\section{RÉSULTATS}

Avant de présenter les résultats de l'inventaire qui ont mis en avant une grande diversité de cas de figures invitant à préciser la notion de «cabanon », il nous semble important de faire un court détour vers l'histoire de l'implantation des cabanons dans la vallée. Celle-ci est en effet étroitement liée à la construction de l'image des lacs de la Sélune comme lieu de pêche et de loisirs.

\section{Les lacs et les cabanons, l'invention des lieux}

La construction du premier barrage hydroélectrique, la Roche-qui-Boit, par la Société des Forces 


\begin{tabular}{|c|c|c|c|c|}
\hline Type de constructions & Pêcheur & Lieu de résidence & Profession & Tranche d'âge \\
\hline $\begin{array}{l}13 \text { usagers et/ou pro- } \\
\text { priétaires de maisons } \\
\text { rencontrés }\end{array}$ & $\begin{array}{l}\text { Oui (1) } \\
\text { Occasionnel (7) } \\
\text { Non (4) }\end{array}$ & $\begin{array}{l}\text { Cantons (8) } \\
+ \\
35(2) \\
14(1) \\
28(1) \\
76(1)\end{array}$ & $\begin{array}{l}\text { Retraités (5) } \\
\text { Employés (4) } \\
\text { Ouvriers (2) } \\
\text { Commerçant (1) } \\
\text { Agriculteur (1) }\end{array}$ & $\begin{array}{l}<25 \text { ans }(1) \\
25-55 \text { ans }(2) \\
>55 \text { ans }(10)\end{array}$ \\
\hline $\begin{array}{l}29 \text { usagers et/ou pro- } \\
\text { priétaires de cabanes } \\
\text { rencontrés }\end{array}$ & \begin{tabular}{|l|} 
Oui (17) \\
Occasionnel (5) \\
Non (7)
\end{tabular} & $\begin{array}{l}\text { Cantons (13) } \\
+ \\
50(5) \\
35(7) \\
14(2) \\
44(1) \\
76(1)\end{array}$ & \begin{tabular}{|l|} 
Retraités (7) \\
Employés (8) \\
Agriculteurs (6) \\
Ouvriers (4) \\
Commerçants (3) \\
Étudiant (1)
\end{tabular} & $\begin{array}{l}<25 \text { ans }(3) \\
25-55 \text { ans }(8) \\
>55 \text { ans }(18)\end{array}$ \\
\hline $\begin{array}{l}9 \text { usagers et/ou proprié- } \\
\text { taires de chalets équipés } \\
\text { rencontrés }\end{array}$ & $\begin{array}{l}\text { Oui (5) } \\
\text { Occasionnel (3) } \\
\text { Non (1) }\end{array}$ & $\begin{array}{l}\text { Cantons (3) } \\
+ \\
35(2) \\
50(1) \\
14(1) \\
\text { Royaume-Uni (2) }\end{array}$ & $\begin{array}{l}\text { Retraités (5) } \\
\text { Employés (3) } \\
\text { Enseignant (1) }\end{array}$ & $\begin{array}{l}25-55 \text { ans }(4) \\
>55 \text { ans }(5)\end{array}$ \\
\hline $\begin{array}{l}2 \text { usagers et/ou proprié- } \\
\text { taires d'abris sommaires } \\
\text { rencontrés }\end{array}$ & $\begin{array}{l}\text { Oui (1) } \\
\text { Non (1) }\end{array}$ & Cantons (2) & $\begin{array}{l}\text { Enseignant (1) } \\
\text { Employé (1) }\end{array}$ & $\begin{array}{l}25-55 \text { ans (1) } \\
>55 \text { ans (1) }\end{array}$ \\
\hline
\end{tabular}

Tableau 2 : Usagers des cabanons rencontrés

Profiles of hut users encountered

Motrices de la Sélune de 1915 à 1919, puis de Vezins de 1929 à 1932, modifie profondément les paysages puisque deux retenues se forment à l'amont de ces ouvrages. Ce changement va rapidement s'accompagner de l'apparition de pratiques récréatives voire touristiques qui restent importantes aujourd'hui (pêche, promenade, etc.). Les lacs fournissent une ressource nouvelle au territoire dont le potentiel est exploité dès les années 1930 : «Ces ouvrages sont, pour l'excursionniste, une agréable surprise en ce qu'au lieu de nuire à la beauté du paysage, ils s'harmonisent avec les charmes naturels d'un site déjà embelli par la vaste nappe d'eau substituée au cours de la rivière »(Ouest-Éclair, 28 mai 1931), peut-on lire dans la presse locale de l'époque. La promotion d'activités comme le canotage témoigne de l'invention des lacs comme lieux attractifs très vite après leur création. Les lacs offrent en effet un certain dépaysement à l'échelle de la Basse-Normandie qui ne comporte pas de telles étendues d'eau et seulement quelques courtes sections en gorges telles la Suisse Normande ou les gorges de la Vire (Brunet, 2001 ; Germaine, 2009). L'attrait du site se traduit par l'installation de divers lieux de divertissement promus par des privés puis par les collectivités locales. À une centaine de mètres du barrage, les ouvriers du barrage venaient jouer aux cartes au "Chalet vert », une guinguette ouverte de 1932 à 1965. De 1934 à 1942, l'Hôtel Bernardin installé au bord du lac de Vezins proposait aux visiteurs des promenades en vedette, en barque ou encore en pédalos. Rebaptisé par ses propriétaires successifs, c'est aujourd'hui un restaurant nommé l'Auberge du lac. En 1964, l'association "Loisirs et Plein Air» établit une petite base nautique au pont des Biards. Le lieu est convivial : les adhérents s'y retrouvent le week-end pour partager un repas, randonner et naviguer sur les lacs. En 1969, un parking et un terrain de camping sauvage sont inaugurés au lieudit «Les petites Bruyères » en rive droite du lac de Vezins. Ce site est utilisé par «Loisirs et Plein Air » de 1968 à 1972. La base déménage ensuite au lieu-dit « la Mazure » en 1975 et change de nom pour devenir la base de loisirs de la Mazure, soutenue par les collectivités locales, qui misent sur le tourisme pour leur développement. Les activités de loisirs s'institutionnalisent et sont promues par les collectivités locales qui tentent de développer une 
destination de tourisme vert autour des lacs du Sud Manche (Germaine et Lespez, 2014). De 1975 à 1992, un spectacle son et lumière mettant en scène des bénévoles se déroule chaque année au bord du lac de la Roche-qui-Boit. Les années 1980 voient s'intensifier l'intérêt des collectivités locales pour les lacs : les locaux de la Mazure sont agrandis à deux reprises et le village de gîtes du Bel Orient situé audessus du lac de la Roche-qui-Boit est ouvert par la commune de Saint-Laurent-de-Terregatte. En parallèle, de nouveaux établissements privés sont ouverts (restaurants, le parc de loisirs l'Ange Michel, etc.) à proximité des lacs. Enfin depuis la mise en service de l'autoroute des estuaires, en 2003, un panneau invite l'automobiliste à prendre la sortie en direction de Saint James pour cheminer sur la « route des lacs du Sud Manche».

La pêche constitue une activité majeure dans la construction de ces lieux. Sa promotion est encouragée par les associations qui procèdent régulièrement à des alevinages et à des travaux d'entretien sur la rivière. Des empoissonnements sont réalisés dès $1932^{4}$. L'augmentation du nombre de pêcheurs créé en effet des besoins croissants d'alevinage : en 1947, la pisciculture de Saint-Hilaire-duHarcouët contenant 30000 œufs de truites fario en incubation devient même insuffisante puis une nouvelle pisciculture est inaugurée en 1957 produisant 800000 alevins. En 1947, une société de pêche est créée spécifiquement autour des lacs : "Association Pêche et Pisciculture Riverains et Pêcheurs Réunis ». Dans les années 1960, elle se divise en plusieurs associations toujours existantes : «la Ducéenne » pour la retenue de la Roche-Qui-Boit et « la truite Saint-Hilairienne » pour Vezins. L'État concède à ces AAPPMA le droit de pêche moyennant une location annuelle versée par les associations ${ }^{5}$. La création des lacs a favorisé la reconversion de la pêche locale vers celle des poissons d'eau calme pratiquée depuis les berges, les ponts mais aussi des embarcations.

4. Le ministère des Travaux publics stipule en 1927 que suite à l'aménagement de la chute de Vezins, et afin de "compenser les difficultés que la présence du barrage apportera aux migrations du poisson et le dépeuplement qui peut en être la conséquence, le concessionnaire fournira chaque année alevins dont les espèces et les quantités seront également indiquées par ce service, sans que toutefois la dépense correspondant à cette fourniture puisse dépasser la somme de $1000,00 \mathrm{~F}$ » (article 7).

5. Depuisle 27 Juillet 1954, EDF a cédé gratuitement les droits de pêche et de chasse à l'État représenté par l'Administration des Domaines des Eaux et Forêts.
La pêche au saumon est désormais cantonnée à l'aval du premier barrage. La pêche à la truite est quant à elle pratiquée en amont du lac de Vezins. La pêche participe fortement à l'animation des lacs à travers l'organisation de concours dont celui organisé des années 1950 à 1987 début juin au pont de Virey (amont du lac de Vezins) qui a réuni jusqu'à 600 pêcheurs (Dodeman, 2008) et de nombreux visiteurs venus profiter des animations accompagnant l'événement (en 1959 par exemple : défilé de chars, fanfare, feu d'artifice, etc.). Si le nombre de pêcheurs a nettement diminué (4200 en 1973, 2500 en 1988, 1100 en 2008 pour «la Truite Saint-Hilairienne $\left.{ }^{6} »\right)$, les lacs restent animés par des manifestations liées à la pêche : ainsi, depuis 2002, le Carp River Club organise des concours de pêche à la carpe ("Enduro carpe », " marathon halieutique », "interclub») une à deux fois par an sur les lacs de la Sélune.

La construction des lacs comme lieu de pêche et de villégiature s'est aussi réalisée au travers l'apparition des premières installations de cabanons sur les berges. La première cabane a abrité des ouvriers travaillant à la construction du pont des Biards dans les années 1930. Mais c'est entre 1955 et 1965 qu'un nombre plus important de cabanes à destination exclusive du loisir est construit aux abords de Vezins, notamment au pont des Biards et sur les rives du Lair. La majorité de celles présentes sur la rive gauche du Lair et le long de l'Yvrande a été construite dans les années 1960. Ce développement est contemporain de la diffusion des pratiques de loisirs au sein des classes populaires et des effets de la mise en place des congés payés dès 1936, qui aura mis près de 20 ans pour susciter un réel accroissement des déplacements de vacances (Richez et Strauss, 1995). Cette période correspond par ailleurs à l'apparition de la "pêche sportive » (Roux, 2007; Corbin, 2009; Barthélémy, 2013). D'autres installations s'effectuent par vague jusque dans les années 1970 toujours en lien avec la pêche même si quelques résidences principales avec vue sur les lacs sont aussi bâties. Les constructions les plus tardives remontent aux années 1990 voire 2000. C'est également dans les années 1990 que plusieurs changements de propriétaires ont lieu : toujours guidés par la pêche, certains de

6. L'adhésion, depuis 2002, de la Manche au principe de réciprocité de l'EHGO - entente halieutique du Grand Ouest - rend difficile l'estimation de la fréquentation des rivières par les pêcheurs. 
ces nouveaux arrivants vont effectuer des travaux considérables de rénovation voire transformer ces bâtiments en résidences principales.

\section{Cabane, bungalow, chalet, maisonnette... ou la nécessité d'une typologie}

169 constructions disposant d'un accès direct aux lacs ont été recensées auxquelles il convient d'ajouter 56 bâtiments annexes (WC, abris de jardin, garage). Face au caractère hétéroclite des constructions rencontrées sur le terrain, il était difficile de déterminer quels bâtiments entraient dans la catégorie des cabanons et lesquels n'en étaient pas : il nous a alors semblé préférable d'élargir l'inventaire à l'ensemble des constructions en bordure des lacs. Cette hétérogénéité renvoie à la diversité de vocabulaire utilisée pour désigner les constructions (cabane, cabanon, chalet, bungalow, abri, construction, maisonnette, préfabriqué, fishing lodge...). Quatre types ont ensuite été distingués en fonction des critères suivants (figure 2 - planche VII) :

- la forme générale de la construction (construction légère ou en dur);

- le niveau de confort estimé par le raccord de celle-ci à au moins un des réseaux de distribution (eau/électricité);

- la légalité de la construction appréhendée par sa mention au cadastre (construction légale ou pas).

Cela permet de distinguer (figure 2 - planche VII) :

- des abris sommaires (15) : abris de pêche situés à moins de $5 \mathrm{~m}$ des berges (parfois sur la berge ou associé à un ponton), présentant une forme « ouverte » et facilement démontables;

- des cabanes (107) : constructions hétéroclites composées de différents matériaux, closes et figurant pour moitié sur le cadastre. Elles ne disposent pas d'un raccord aux réseaux mais d'autres éléments de confort (poêle à bois, systèmes de batteries de $12 \mathrm{~V}$ ou de génération d'électricité pour s'éclairer ou faire fonctionner des équipements électriques);

- des chalets équipés (18) : bâtiments disposant d'un raccord aux réseaux d'eau et/ou d'électricité mais ne disposant pas de murs en dur ou non recensés sur le cadastre ${ }^{7}$. Ces constructions pré-

7. Deuxconstructions de cette catégorie présentent la particularité d'être raccordées au réseau EDF sans que le bâtiment ne soit présent sur le cadastre. sentent un niveau de confort supérieur aux cabanes (antenne TV, dispositif de ventilation, boite à lettres) et l'un d'entre eux est habité à l'année;

- des maisons (29) : bâtiments classiques d'habitation, cadastrés, raccordés au réseau électrique et à celui d'eau potable. 12 d'entre elles sont occupées à l'année.

L'inventaire révèle que les cabanes et chalets sont prédominants parmi les constructions disposant d'une vue ou d'un accès aux lacs.

\section{La répartition des constructions : avoir les pieds dans l'eau ou une vue sur les lacs?}

Les constructions ne sont pas réparties de manière uniforme autour des lacs mais concentrées sur le lac de Vezins, plus spécialement sur le Lair et en rive gauche de l'Yvrande (figures 3 et 4 planches VIII-IX). Leur localisation répond à différents éléments : l'accessibilité (proximité de ponts, d'une route ou a minima d'un chemin), la topographie et la nature du site (pente, occupation du sol) et la possibilité juridique de s'y établir (la majorité des berges du lac de la Roche-qui-Boit appartient à EDF ce qui limite les possibilités de s'établir). Les maisons et chalets équipés occupent majoritairement les points hauts qui offrent à leurs occupants une vue sur les lacs tandis que les cabanes sont directement au bord de l'eau. On retrouve ici la distinction repérée par A. Maugin et S. Perault (2011) sur les bords de l'Oise entre ceux qui sont dans la nature et ceux qui donnent sur la nature. Par ailleurs, les maisons et chalets équipés sont desservis par une route carrossable tandis que les cabanes sont plus souvent éloignées de la route : la moitié n'est pas accessible par une route carrossable, il faut emprunter un chemin de terre, souvent privé, ou bien laisser la voiture à distance et rejoindre à pied la cabane. Les cabanes sont donc situées pour la plupart en bordure de lac dans le sous-bois, et donc partiellement cachées à la vue.

Elles sont toutes reliées à l'eau par un ponton ou une plateforme maçonnée : il s'agit d'accéder aux zones de pêche pour la plupart des usagers $(66 \%$ se définissent comme étant pêcheurs et plusieurs autres admettent s'adonner occasionnellement à cette pratique) ou bien à l'eau pour ceux qui ne sont pas pêcheurs mais recherchent la proximité des lacs. 


\section{Discussion}

Les enquêtes révèlent que les cabanons et les lacs évoquent des atmosphères très contrastées : d'un côté des pratiques contemplatives de la nature, solitaires, en duo ou en famille, et d'un autre côté des usages collectifs (en petit groupe d'hommes le plus souvent) conviviaux (parties de pêche) voire festifs des lacs. Ces deux ambiances, souvent décrites par les mêmes personnes, ne sont pas spécifiques à la Sélune comme le note B. Picon (2001) : « La cabane a aussi vocation à combler deux désirs opposés, la convivialité et la solitude. »

\section{Le cabanon ${ }^{8}$, un rapport individuel privilégié à la nature}

\section{Auto-construction et liberté architecturale}

L'inventaire met en évidence l'hétérogénéité des constructions. Au-delà de la diversité de matériaux, de formes, de couleurs ou encore de styles, l'ensemble des constructions, hormis les maisons, partage la particularité d'utiliser des éléments bon marché (bois, tôle ondulée, plaque de ciment) ou de récupération. L'auto-construction et le bricolage dominent. L'astuce et l'inventivité sont la règle. G. Raveneau et O. Sirost (2011) parlent de «villégiature expérimentale dans les marges de la tradition balnéaire ». Un soin est apporté à l'entretien et à la personnalisation des lieux. Si les matériaux utilisés sont peu onéreux, ils font l'objet d'améliorations et de personnalisations originales : poteau FranceTélécom utilisé pour la structure d'un ponton, poteau EDF en guise de jardinière, ancienne barque pour ranger le bois (figure 5). Les cabanons sont construits, entretenus et améliorés par séquences au fur et à mesure qu'ils nécessitent d'être consolidés. Certaines parmi les premières cabanes n'ont d'ailleurs été que l'acte d'un premier développement (Loubes, 2001) vers la construction d'un chalet ou d'une résidence secondaire (Perrot et la Soudière, 1998). Ces cabanons ressemblent en cela à ceux décrits par N. Ortar (2001) : "l'une des caractéristiques [...] est l'absence de plan d'architecte et de souci d'intégration à un style architectural particulier ». Ainsi, le paysage est marqué par un caractère très hétéroclite et se démarque des normes officielles

8. Nous entendons par « cabanons » l'ensemble des abris, cabanes et chalets. de construction. Les cabanons de la Sélune se distinguent de ce point de vue des abris standardisés qui s'imposent de plus en plus dans les lotissements pavillonnaires ou les jardins familiaux (Cabedoce et Pierson, 1996).

Les occupants se préoccupent plutôt d'élaborer un lieu qui réponde à leur pratique, essentiellement la pêche, mais pas seulement, et ils aménagent ce lieu en conséquence. L'intérieur est souvent spartiate. On y trouve le matériel nécessaire à la pêche : des cannes, des seaux, des sièges, un réchaud et quelques éléments d'épicerie, quelques meubles abritent un peu de vaisselle, et souvent, un lit est installé au fond du cabanon. Les espaces extérieurs font également l'objet d'une certaine attention : l'accès aux berges, parfois distantes de quelques dizaines de mètres, est aménagé (sentier, marches, rambardes). Les abords de nombreuses constructions, parmi les cabanes et chalets équipés, sont fleuris voire jardinés et les haies entretenues (figure 5). Tables et chaises occupent les abords des cabanons souvent terrassés afin de profiter au mieux de la vue sur le lac. Des tentes sont parfois aussi installées pour accueillir des amis supplémentaires le temps d'une partie de pêche. Camouflés parmi la végétation, les cabanons sont entourés de bois qui sont entretenus voire utilisés (bûches débitées) et dans lesquels on devine quelquefois la construction d'une cabane perchée.

\section{En quête de nature}

Au-delà de la pêche, le cabanon est avant tout le vecteur d'un retour vers la nature. Venir au cabanon, c'est avant tout la possibilité de vivre, au moins temporairement, dans un cadre dans lequel on peut directement pratiquer des activités proches de la nature (pêcher, ramasser des champignons, observer les animaux, naviguer sur les lacs, etc.) et profiter des paysages. La pêche est d'ailleurs souvent mise au second plan, même par des pêcheurs passionnés, pour mettre en exergue le plaisir d'être ici : les lacs permettent de revenir à des "plaisirs simples». La cabane est perçue comme un lieu d'éveil des sens. Les propos des occupants sont riches en description des couleurs de certains sites à l'automne notamment. Beaucoup d'entre eux mettent en avant la possibilité de voir de nombreux animaux : «quand je passe au pont des Biards, systématiquement je ralentis 


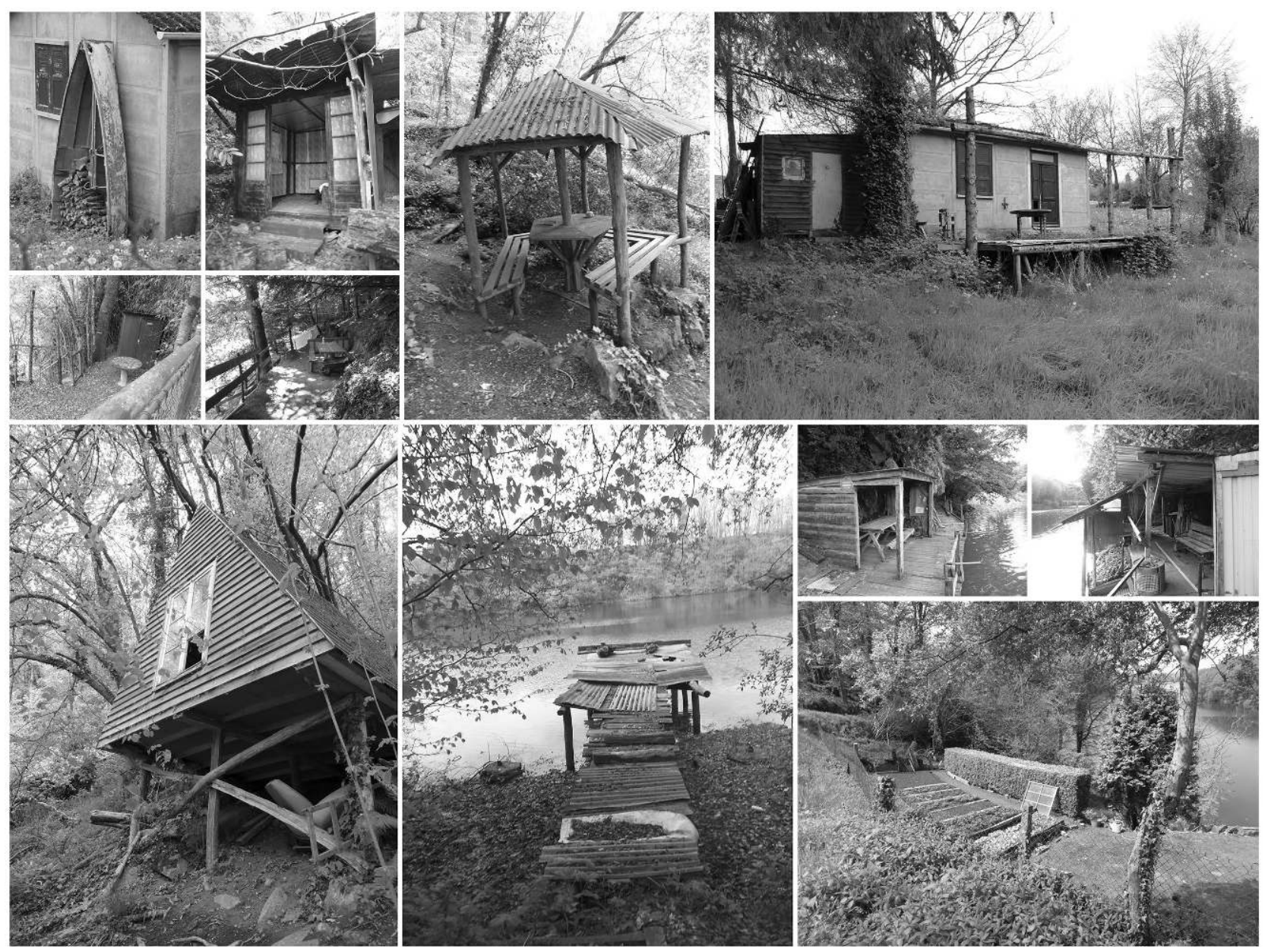

Figure 5 : Illustrations de la liberté de construction au bord de la Sélune Examples of architectural freedom in lakeside constructions

et je regarde. J'aime voir les mouettes, j'aime voir les cormorans, j'aime voir... même un rat musqué. J'aime la nature moi. Mais je l'aime comme elle est là : avec son lac» (propriétaire d'une résidence principale, 70 ans) ou «ce qui est beau à voir ici pour celui qui veut y assister, c'est quand les carpes frayent. Elles ont le dos qui sort de ça de l'eau, ça patouille là-dedans, c'est des centaines et des centaines de carpes qu'il y a hein! Parce qu'il y a des herbes alors elles viennent pondre là-dedans... Ah, si, c'est extra! » (propriétaire d'un chalet équipé en bordure du lac, 70 ans). Les usagers témoignent d'une connaissance très fine du terrain et de l'écosystème des lacs qui illustre leur préoccupation vis-à-vis de la nature : ce lien à la nature ne passe pas que par la prédation par la pêche (ou la chasse) mais aussi par son observation. Plusieurs personnes nous ont montré des photos d'animaux ou de plantes observés auprès des lacs comme la lathrée clandestine par exemple. Si les animaux observés ne correspondent pas aux espèces emblématiques de la biodiversité recherchée par les gestionnaires, les qualificatifs employés par les usagers des cabanons montrent qu'ils considèrent l'espace des lacs comme «sauvage » et «naturel». Ils sont très attachés à ce caractère qui participe de l'attrait des lieux. Le caractère artificiel des lacs n'est jamais évoqué et jamais perçu comme pénalisant. Les usagers mettent à l'inverse en avant la présence de rochers, de bois, d'étendues d'eau qui symbolisent la nature.

Paradoxalement, la vie dans la nature qui est recherchée à travers la cabane ne fait pas l'économie d'un nécessaire entretien du lieu. L'appropriation matérielle de l'espace se traduit par le nettoyage des abords des cabanons et la mise en ordre de la nature à proximité. Ces aménagements, rappelés par 
la majorité des occupants rencontrés, apparaissent cohérents avec les attentes qu'ils formulent en termes de valorisation et d'entretien de la vallée, où domine la crainte d'une vallée délaissée et laissée à l'abandon. S'ils apprécient son caractère « sauvage », beaucoup s'inquiètent de savoir qui va assurer l'entretien de la vallée afin qu'elle ne se transforme pas en une immense «friche ». Le cabanon s'inscrit ainsi comme un espace de transition entre la nature et la culture : il offre un rapport privilégié à la première mais constitue également un refuge (Sirost et Raveneau, 2011). Cela tend aussi à montrer en quoi cette nature, qui est recherchée, doit être aussi «sauvage » que « sociale».

Cette quête de nature est associée au caractère calme et reculé des lieux largement mis en avant par les personnes rencontrées : "on est tranquille là, c'est le calme: Dans le temps, j'avais un commerce et tout ça, je venais le dimanche : c'était... c'était reposant» (propriétaire d'un chalet équipé, 77 ans). Le cabanon constitue un refuge, un microunivers à part qui permet de s'échapper de la ville et de ses nuisances. «Le plaisir de sortir de la ville» est en effet invoqué, de même que le calme et l'absence de bruit "pour ceux qui bossent à l'usine». Les gorges de la Sélune constituent un des multiples lieux dans lesquels s'impliquent les individus enquêtés : bien qu'habités temporairement et distants des lieux de travail et de domicile, les cabanons constituent un lieu récréatif approprié par des individus caractérisés par une certaine mobilité spatiale (Stock, 2006). La tranquillité et la sérénité des lieux permettent d'accéder à tout un panel de sons : échos d'une voix dans une vallée silencieuse, carpes qui frayent, bourdonnement d'insectes, cris et chants d'oiseaux, vaguelettes occasionnées par une embarcation, etc. Les cabanons offrent d'autant plus un repos de qualité qu'ils ne sont pas facilement accessibles : il faut connaître les lieux pour y venir et il y a peu de signalétique. Cela se mérite en quelque sorte (Claeys-Mekdade, 2001). Cela renforce le caractère secret et tranquille du lieu. L'absence de réseau de téléphonie mobile performant et la faible fréquentation du site confèrent un caractère isolé à cette partie de la vallée. Séjourner ici permet de goûter au plaisir d'être légèrement coupé de la civilisation. Ces lacs jouent même pour certains une fonction thérapeutique : ils mettent en avant leur bonne santé malgré leur âge, conser- vée selon eux grâce aux bienfaits du site. Les noms donnés à certaines cabanes ainsi que les plaques fixées aux bâtiments témoignent de cette double qualité, naturelle et tranquille, recherchée par les usagers : Le repos du pêcheur, Le beau vallon, Tout près du lac, Le bout du monde, la maison des écureuils, etc. Ce rapport au cabanon en lien avec la nature et la recherche de tranquillité est en effet avant tout individuel, au mieux familial.

Au final, peu de choses déplaisent aux usagers dans cet environnement, et s'il en existe, ce sont en général les autres : "les gens dégueulasses qui laissent leurs ordures ", les jeunes en barques qui font du bruit, les vols et les actes de vandalisme, et du côté de la nature, ce qui prolifère de trop comme les ragondins et les silures. Ainsi, l'environnement et le cabanon s'apprécient tels quels et apportent tout ce qui semble nécessaire à une vie épanouie : "Il fait beau, on a une tonnelle. On met la tonnelle ici. Je mets les piquets là pour attacher. Qu'est-ce que vous voulez-de plus?»

\section{Un attachement familial}

La majorité des usagers interrogés réside dans un périmètre proche des lacs puisqu'ils habitent un canton riverain pour $65 \%$ d'entre eux ou se concentrent dans le reste du département et dans la région de Fougères en Ille-et-Vilaine pour $28 \%$ (tableau 2). Si certains viennent de plus loin, il est fréquent qu'ils soient originaires de la vallée ou qu'ils y aient des liens familiaux. L'attachement aux lieux s'effectue aussi à travers une histoire familiale qui s'écrit grâce aux cabanons. Ils se transmettent de génération en génération comme d'autres auteurs l'ont observé ailleurs (Claeys-Mekdade, 2001 ; Nicolas, 2008). C'est d'ailleurs en partie cette charge affective qui rend si difficile l'évaluation monétaire de ces biens. Pour les propriétaires, la valeur des cabanons ne saurait se résumer au prix du terrain et de quelques planches de bois tant ils représentent le travail d'aménagement fourni depuis plusieurs années voire toute une vie ainsi que le confort qui en est retiré.

La construction de la cabane, son entretien et l'aménagement du terrain semblent être des activités réservées aux hommes de la famille. Dans certaines cabanes disposant d'un niveau de confort élémentaire (générateur d'électricité, possibilité de couchage correct, taille supérieure du bâti- 
ment) ainsi que les chalets équipés, il n'est pas rare qu'après un temps exclusivement masculin (pêche, remise en état du terrain, repos solitaire) suive un temps où sont présents femmes et enfants le temps d'une journée ou d'un week-end. Ces séjours donnent alors lieu à d'autres types d'activités : promenade (jusqu'au pont, en vélo, en barque), sortie dans les environs (bois voisins, parc de loisirs de l'Ange-Michel, base nautique de la Mazure, etc.). Le cabanon est donc un lieu privilégié de réunion familiale mais aussi de transmission de savoirs entre les générations que ce soit à travers les activités de bricolage inhérentes à l'entretien du site, d'observation de la nature ou de pêche.

Beaucoup des personnes rencontrées sont âgées (80\% ont plus de 55 ans) et ont gardé la mémoire des cabanons depuis plusieurs dizaines d'années. Aujourd'hui retraitées, une partie de leurs souvenirs d'enfance sont reliés à ces cabanons et au lac (tableau 3). Elles se remémorent les vacances passées dans la cabane des grands-parents ou des parents, les parties de pêche et les premiers bains dans les eaux du lac où elles ont appris à nager. L'appropriation des lieux s'opère donc par un apprentissage et une familiarisation, le plus souvent assurés par la sphère familiale, qui permet d'acquérir les bonnes pratiques. Il existe ainsi une toponymie locale qui permet d'identifier des lieux de pêche mais aussi de se repérer pour naviguer sur les lacs. Celle-ci est transmise aux enfants ou petits-enfants en même temps que les histoires associées à différents lieux aussi bien sur les rives (rocher, point de stockage d'appâts, etc.) qu'au milieu des lacs (ancien pont, ancienne route, souche d'un arbre, etc.).

\section{Les bords des lacs, lieux d'une sociabilité exclusive}

\section{Lieux de sociabilité masculine}

Si le rapport au cabanon est d'abord individuel et familial, les occupants viennent aussi auprès des lacs entretenir certaines formes de sociabilité, le plus souvent organisées autour de la pêche. La majorité des cabanes, dont la vocation principale est la pêche et dont le niveau de confort est assez sommaire, n'est fréquentée que par des hommes. La présence des femmes est néanmoins plus courte que celle des hommes et aucune femme n'a été rencontrée seule dans les cabanons. Ils pêchent les carnassiers, le «blanc », ou encore la carpe, souvent en groupe, et avec une dimension sociale importante : "Le lac, c'est les copains, si j'avais été tout seul aujourd'hui, je serai allé à la truite. » La cabane représente avant tout le point de départ et de retour de nombreuses parties de pêche : c'est ici que sont conservés les vifs et du matériel et que s'additionnent parfois sur les murs les photos de belles prises. La pêche pratiquée est présentée comme sportive et ludique : c'est le plaisir d'arriver à sortir un gros poisson qui est ensuite relâché et de nouvelles pratiques chez les jeunes qui apprécient par exemple le «fun » de pêcher en float-tube. La cabane est aussi décrite comme un lieu de convivialité (Nicolas, 2008) : c'est la bande de copains qui vient pêcher mais aussi faire un barbecue au cabanon ou boire l'apéritif. Le cabanon génère une activité de groupe (Brun et al., 2001). Les occupants s’y retrouvent à plusieurs : l'agriculteur voisin s'arrête boire le café, les enfants ou les amis peuvent passer... Ces lieux sont aussi l'occasion de venir faire la fête, l'isolement des lieux autorisant à peu se soucier
Tableau 3 : Extraits d'entretiens auprès de propriétaires de cabanons

Excerpts from interviews with hut owners

\begin{tabular}{|c|c|}
\hline $\begin{array}{l}\text { êt pếcher.... Mon fils serait là, ce serait la même [...] il est venu avec des copains, il } \\
\text { ait des parties de pêche, ils pêchaient la nuit, ils s'amusaient. voilà on est au calme } \\
\text { est tranquille. Mais la pêche c'était son truc aussi et puis voilà, disons qu'ils ont été } \\
\text { rcés là-dedans mes enfants, comme moi j'ai été bercé là-dedans aussi, voilà. Et là } \\
\text { tait mon petit-fils qui est bercé là-dedans. » }\end{array}$ & $\begin{array}{l}\text { Propriétaire d'un } \\
\text { chalet équipé et de } \\
\text { cabanes ( } 52 \text { ans) }\end{array}$ \\
\hline $\begin{array}{l}\text { "Moi je suis de la région fougeraise, je vena } \\
\text { alors! Hein j'ai } 77 \text { ans ! On venait à la pêc } \\
\text { vère. ... là depuis } 2 \text { ou } 3 \text { ans j'ai mon fils qui }\end{array}$ & $\begin{array}{l}\text { Propriétaire d'un } \\
\text { chalet équipé ( } 77 \\
\text { ans) }\end{array}$ \\
\hline $\begin{array}{l}\text { En fait } \\
\text { errain ils } \\
\text { ur Saint }\end{array}$ & $\begin{array}{l}\text { Copropriétaire } \\
\text { d'une maison et } \\
\text { d'une cabane sur le } \\
\text { lac ( } 29 \text { ans) }\end{array}$ \\
\hline
\end{tabular}


du volume de la musique et des discussions, tout en ayant un terrain suffisamment grand pour accueillir d'éventuels tentes ou hamacs pour héberger des invités. Situés à l'écart, ces lieux peuvent accueillir des activités à la marge du comportement socialement admis : on y vient faire du bruit, consommer de l'alcool... Ces pratiques sont plus généralement développées sur le lac de Vezins plus fréquenté que le lac de la Roche-qui-Boit mais il n'existe pas de différenciation claire des pratiques des lieux autour de ce lac.

\section{Des arrangements entre voisins et avec les propriétaires fonciers}

La sociabilité liée aux cabanons génère une organisation propre à l'échelle des « quartiers » de cabanons. Des réseaux de voisinage se dessinent au travers des entretiens réalisés. Les propriétaires et usagers sont liés par un certain nombre d'arrangements autour de l'utilisation des pontons et même des cabanes qui peuvent être prêtés. L'échange se fait parfois en contrepartie de menus services : l'entretien du jardin ou quelques travaux. Le statut temporaire de l'occupation oblige également les usagers à s'organiser entre eux pour surveiller qu'il n'y ait pas de vol ou de dégradation durant leur absence. La villégiature à proximité du lac représente aussi pour certains une forme de responsabilité vis-à-vis du site : ils se doivent de veiller à son entretien au fil des années afin de pouvoir continuer d'y venir. Sur la plage de Piémanson, C. Claeys-Mekdade (2001) remarque des discours similaires de la part des campeurs qui mettent en avant leur rôle dans la protection de la nature (des dunes). Des chantiers de débroussaillage ont d'ailleurs été organisés par l'association des Amis du Barrage, qui défend le maintien des lacs, en mars 2015 réunissant une soixantaine de bénévoles parmi lesquels figuraient des usagers des cabanons.

Les relations sociales qui se nouent autour des cabanons font intervenir d'autres acteurs lorsque les cabanons sont construits sur la propriété d'une tierce personne. Près de $60 \%$ des constructions figurent au cadastre : les absentes renvoient aux plus légères et à des aménagements démontables. Sur les terrains privés, l'usager peut être propriétaire du cabanon et de la parcelle, propriétaire du cabanon et louer le terrain ou encore louer le cabanon au propriétaire. Si la construction est déclarée, une transaction financière peut exister entre l'usager et l'occupant. Par exemple, le secteur du pont des Biards appartient à un agriculteur, très impliqué dans la société de pêche, qui loue à la fois des terrains et des cabanes contre rémunération tandis que la rive gauche du Lair appartient, elle, à un seul propriétaire qui ne demande pas de contrepartie financière. La proximité des parcelles agricoles donne lieu à d'autres arrangements. Ainsi, il n'est pas rare qu'un propriétaire de terres riveraines s'arrange pour faciliter la pratique de la pêche en donnant la permission aux propriétaires de cabanes d'utiliser un chemin qu'ils entretiennent, baisse ses clôtures pour laisser le passage ou ne clôture pas jusqu'au cours d'eau pour faciliter l'accès. Un agriculteur possédant une parcelle riveraine sur laquelle sont implantées des cabanes explique: "L'accord était simple, mon père louait le terrain, autorisait la construction de cabanes et les surveillait en l'absence des propriétaires. » Le cabanon a donc un statut juridique et une existence sociale flous.

Avant la construction des barrages, les propriétés privées allaient jusqu'à la rivière. L'État (propriétaire du barrage de Vezins) et EDF (exploitant des deux barrages et propriétaire du barrage de la Roche-quiBoit) sont devenus propriétaires d'une partie du linéaire des rives du lac de Vezins et de l'essentiel du linéaire du lac de la Roche-qui-Boit. Certains usagers ont donc aussi des relations avec l'exploitant des barrages. Aucun chalet ou maison n'est présente sur le domaine d'EDF mais 8 abris sommaires et 12 cabanes y ont été recensés ainsi que la majorité des pontons dont les pieds sont fixés avec du béton dans le lac. Une partie seulement de ces constructions s'inscrit dans le cadre d'une convention ${ }^{9}$ avec EDF témoignant d'une certaine tolérance de l'entreprise. Une partie des constructions semble avoir été réalisée sans grande préoccupation du statut foncier comme en témoigne cette propriétaire : " mais tu n'as pas le droit de faire ça" a-t-elle dit à son père qui s'est construit une cabane sous le pont de la République et celui-ci lui a répondu «ah bon, c'est chez moi ici ». Cette anecdote tend à montrer que l'appropriation des lieux s'est réalisée indépendamment du statut foncier de l'espace.

\footnotetext{
9. L'entreprise EDF a mené en 2011 un inventaire des cabanons sur le domaine public concédé visant à mettre à jour les conventions établies entre l'entreprise et les propriétaires de cabanons (Terragone et Sevaux, 2011) : celui-ci recense 167 constructions - essentiellement des pontons - dont 46 seulement font l'objet d'une convention.
} 


\section{Modes d'appropriation des berges : une forme de privatisation/accaparement des berges?}

La forte appropriation des lieux par les occupants des cabanes se matérialise dans l'espace par l'aménagement de clôtures, fils barbelés, grillages qui marquent les limites de chacun et de portails parfois imposants par rapport aux constructions auxquelles ils donnent accès (figure 6). Le marquage important de l'espace par de nombreux panneaux d'interdiction témoigne par ailleurs de la volonté des occupants d'interdire aux visiteurs l'accès aux cabanons. Les portails sont tenus cadenassés y compris lorsque l'usager est présent afin d'éviter les intrusions (vols) et le dérangement. Quelques inscriptions plus franches - chien méchant, zone piégée, cabane piégée - tentent de décourager les plus audacieux. Les usagers des cabanons expliquent prendre ces dispo- sitions pour éviter que des méfaits soient commis en leur absence (utilisation de leurs aménagements/ matériels, vol, vandalisme, squat).

Ces signes décrits dans d'autres lieux constituent un des repères de la privatisation des berges (Féménias et al., 2011) ou du littoral (Minvielle, 2005) largement dénoncée. La présence des cabanes contraint de fait l'accès et la circulation le long des lacs. Ce problème est dénoncé par certains usagers comme l'AAPPMA « la Ducéenne » qui revendique un accès aux berges actuellement difficile. Du fait que les propriétaires des berges, l'État et EDF ont cédé leur droit de pêche aux AAPPMA locales, les rives devraient être accessibles aux pêcheurs. Cela n'est le cas que pour une partie d'entre elles. En rive gauche de l'Yvrande et le long d'une partie des rives du Lair, les cabanoniers prennent soin de laisser un passage. Il arrive toutefois de devoir remonter vers
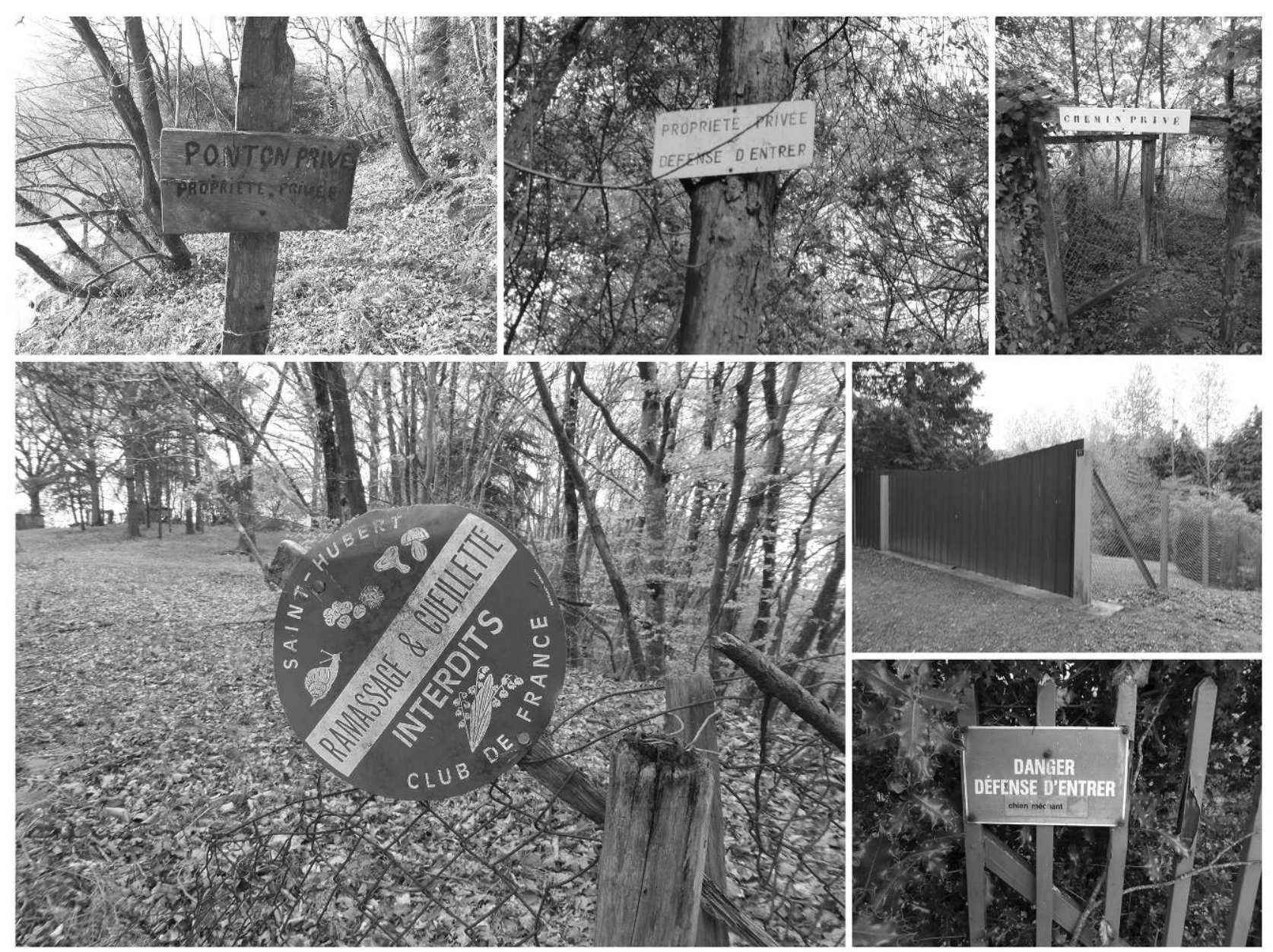

Figure 6 : Illustrations des signes de privatisation Examples of privatization 
la route ou rebrousser chemin face à une clôture. Les accès étant rares et confidentiels, la possession d'un cabanon et/ou d'un ponton mais aussi d'un bateau ${ }^{10}$ est considérée comme un privilège pour accéder aux lacs. Les autres doivent se contenter des ponts et des quelques pontons publics ${ }^{11}$ tandis que le manque d'entretien des chemins réduit l'accès aux bords des lacs pour les promeneurs. Cet accaparement des lacs est, pour certains, responsable d'une faible diversification des activités récréatives. Ce constat a d'ailleurs parfois débouché sur l'expression de regrets : "si on avait eu des aménagements, nos barrages n'auraient peut-être pas été la cible» (un riverain); "si on avait su mieux le valoriser et aussi mieux le partager, nous les pêcheurs, peut-être qu'on n'en serait pas là » (un responsable d'association). La reconstitution de la trajectoire de ces lieux révèle une concurrence entre les usagers des lacs. Les pêcheurs ont en effet empêché le développement d'autres activités récréatives dans les années 1980 en réclamant l'interdiction de la navigation au-delà d'une certaine vitesse, ce qui a mis fin à la pratique du ski nautique un temps proposée sur le lac de Vezins. Les pêcheurs ont donc œuvré pour conserver un usage exclusif des lacs. Ces difficultés d'accès et de cheminement peuvent toutefois être relativisées puisque rien ne garantit que l'accès aux lacs soit plus aisé sans les cabanes, leur présence étant le plus souvent garante d'un entretien minimum du pourtour des lacs. La situation est d'ailleurs identique le long de la rivière qui est dans sa majeure partie non domaniale et inaccessible : l'aval de Ducey se distingue en revanche par l'aménagement d'un sentier et de pontons handi-pêche permettant d'approcher la rivière. Ainsi, si les cabanons sont le support d'une sociabilité riche, celle-ci semble dessiner un usage exclusif des berges et des lacs qui laisse de côté les autres usagers.

10. Parmi les occupants de cabanons rencontrés, tous disent disposer d'une embarcation tandis que c'est seulement la moitié des pêcheurs non occupants de cabanon.

11. En 1993, à l'occasion de la vidange, 5 pontons publics en bois ont été aménagés par l'Association de développement des lacs et du bocage du Sud Manche sur le lac de Vezins (la Mazure, pont des Biards, lieu de pêche de la Chandelle, pont de la République, ancien camping sauvage de St-Martin-de-Landelles) en raison de la forte fréquentation mais aussi pour satisfaire les AAPPMA locales qui réclamaient plus de postes de pêche. Il existe également 3 plateformes maçonnées dans la partie aval du lac de Vezins (Petit Bois d'Isigny) et 6 zones de mises à l'eau publiques accessibles en voiture sur les deux lacs.

\section{Les lacs dans le territoire}

\section{Un faible intérêt des pouvoirs publics pour les cabanons}

On peut s'étonner du faible intérêt qu'ont suscité ces constructions dans les réflexions qui accompagnent la mise en œuvre d'un nouveau projet pour la vallée. Leur présence est mentionnée dans le diagnostic paysager rendu par le cabinet Etheis (2012) à l'occasion de la rédaction du schéma de développement durable pour la vallée commandé par la DDTM de la Manche mais elle n'apparaît pas dans les différents documents présentés par le groupe Maîtres du Rêve (associant pourtant une équipe de paysagistes) mandaté par le Syndicat du Pays de la Baie pour définir le projet de valorisation socio-économique de la vallée de la Sélune après l'effacement des barrages. Les prises de parole des élus locaux lors des réunions publiques ou des comités de pilotage laissent deviner qu'ils négligent cette question car ils considèrent que c'est un problème mineur du fait de l'état d'abandon avancé de la plupart des constructions : pour eux, seules quelques constructions (parmi les chalets équipés) méritent d'être maintenues. D'autres associent la présence des cabanons à la privatisation des lacs en déclarant qu'il s'agit d'une question limitée à quelques familles pendant que la majorité de la population est privée d'accès aux lacs : "il y avait mieux à faire " (un maire). Cette faible prise en compte est d'autant plus étrange que la zone ennoyée devrait être cédée par EDF à l'État qui confiera les 200 hectares libérés aux collectivités locales après la suppression des barrages. Si l'entreprise a lancé un inventaire des constructions établies sur sa concession, aucune information n'est donnée sur le devenir des conventions. Le rapport de la mission de contre-expertise réclamée par la ministre Madame Ségolène Royal (Perrin et al., 2015, p. 95) pointe, lui, cette question : "À certains endroits [...], des pontons et des bungalows ont été installés sur les rives par des propriétaires voisins, en partie sur le domaine public. Bien que la mission ne se soit pas livrée à un décompte précis, elle souligne qu'un nombre important de ces installations ne dispose pas d'une autorisation d'occupation temporaire du domaine public en bonne et due forme. Beaucoup sont par ailleurs en mauvais état. Les accès aux rives sont 
difficiles et les dégagements visuels encombrés. » Les pouvoirs publics semblent ainsi restreindre leur analyse au seul domaine public, qu'ils devront in fine gérer et aménager, mais cela revient à ne s'intéresser qu'aux pontons et donc à minimiser l'ampleur des bâtiments concernés.

Comme dans d'autres cas, les cabanons sont en fait appréhendés comme des " points noirs paysagers ». Bien qu'il traite de thématiques environnementales techniques (Dossier d'autorisation au titre de la loi sur l'eau et de la mise en conformité des documents d'urbanisme), le dossier d'étude d'impact soumis à enquête publique à l'automne 2014 mentionne à 7 reprises les pontons et 6 fois les termes cabane/cabanon. Il est indiqué que " des installations de pontons et cabanons en pourtour des lacs sont présentes et représentent un patrimoine propre à l'état actuel des retenues. Ces installations seront directement impactées par la disparition des plans d'eau » (p. 40). Cette question est également traitée d'un point de vue esthétique : "Plusieurs points noirs paysagers peuvent être mis en avant : le développement de friches autour des pontons qui ne seront plus connectés à la rivière, et plus encore sur les terrains occupés par des résidences secondaires hétéroclites (qui échappent à la gestion publique du fait du caractère privé du foncier) ou encore l'intégration paysagère des infrastructures telles que les ponts (République, Biards) qui deviendront disproportionnés par rapport à la taille de la rivière. » (p. 102). Il s'agit en fait de phrases reprises du Schéma de développement durable de la vallée de la Sélune remis par le cabinet Etheis à la DTTM de la Manche en 2012 auxquelles a été ajouté un jugement de valeur négatif.

Les cabanons présents autour du lac de Vezins ne sont donc pas reconnus par les acteurs institutionnels et politiques comme faisant partie du patrimoine des lacs. Pour les élus des communes riveraines des lacs qui ont été rencontrés, ces cabanons sont liés aux lacs et à l'activité de pêche : selon eux, ils n'ont plus de sens si les lacs disparaissent. Ils font le constat qu'une partie est abandonnée. Certains évoquent par ailleurs leur caractère illégal. Ainsi, pour un des élus interrogés, réaménager la rivière passera par l'enlèvement d'une partie des constructions : "Il y a quelques maisons qui méritent d'être conservées, mais les trois quarts c'est des cabanes, c'est... c'est... voilà. Donc il y a un gros travail aussi à faire. » Un autre élu se questionne sur la restriction de l'usage des bords de lac par quelques familles, celles ayant accès à un cabanon. Si la question du maintien d'éléments bâtis au titre de la conservation du patrimoine a fait l'objet de débats dans les comités de pilotage, elle est réduite aux voutes du barrage de Vezins et aux bâtiments d'exploitation des deux barrages. Comme le constate B. Picon, « hormis en Afrique ou au Japon où elle a souvent un sens sacré, la cabane choque les convenances établies parce qu'elle n’est en conformité avec aucun schéma établi, avec aucune esthétique reconnue, avec aucune tentative patrimonialisante » (2001, p. 335). La comparaison avec d'autres lieux montre que c'est le plus souvent d'une manière négative et sous le terme de "cabanisation » que ces constructions sont évoquées : elles sont dénoncées comme des points noirs paysagers (PNR Oise, 2008). La lecture des Atlas de paysage rédigés à l'échelle départementale ou régionale en France est à ce titre évocateur : la cabanisation y est le plus souvent évoquée comme un point noir à résorber à propos par exemple des Marais de la Souche ou la vallée de l'Aisne (Aisne, 2004), la vallée de la Somme (Atlas des paysages de la Somme, 2007) ou du Thérain (Atlas des paysages de l'Oise, 2005). Des recommandations sont faites afin d'« harmoniser l'architecture ou les couleurs des cabanons de pêche » à proximité des plans d'eau (Atlas des paysages de la Nièvre, 2011). Ces aménagements parfois qualifiés de « sauvages » sont désignés comme participant au mitage du paysage et à la déqualification des espaces (Atlas des paysages de la Sarthe, 2005). La mise en place de la loi Littoral dès 1986 et l'émergence de nouveaux zonages environnementaux ont conduit à la réalisation d'inventaires de ces habitats temporaires (en bordure de plans d'eau littoraux en Languedoc-Roussillon ou au sein du PNR Oise Pays de France par exemple). Ces démarches, entreprises par des administrations de l'État ou les collectivités territoriales ont des finalités opérationnelles, la lutte contre le phénomène de « cabanisation » en vue de sa résorption (Cadoret, 2006; Brun et al., 2001; Geindre et al., 2009). Cette position dominante des paysagistes et des administrations de l'État peut expliquer le désintérêt des pouvoirs publics pour le devenir des cabanons. Il s'agit alors juste de déterminer les modalités de leur démantèlement pour assurer une mise en sécurité du site. 


\section{Vers un abandon des cabanons?}

Malgré un fort attachement aux cabanons, un quart d'entre eux présente un aspect abandonné. Ce constat partagé fait écho aux souvenirs nostalgiques liés à la vie du lac et à un âge d'or qui semble désormais révolu. À qui la faute? À la disparition progressive des cabanoniers et un désintérêt de la part des plus jeunes? À la diminution des poissons dans le lac, à la prolifération des silures, à une évolution des pratiques de loisir ou bien à l'annonce de l'arasement des barrages? La survie des cabanes est suspendue à la décision d'arasement. Leur temps est compté. Certaines commencent d'ailleurs à être désertées. Sans lacs, ces cabanons n'ont pour la plupart de leurs occupants aucune raison d'être, perchés qu'ils seront à 20 ou $30 \mathrm{~m}$ au-dessus du lit de la rivière. Des usagers décrivent leur crainte du grand trou qui les attend, ils expriment une peur majeure, celle de la friche à venir, pour eux synonyme de mort. Cette propriétaire d'un cabanon craint «le trou géant qu'il va y avoir». De nombreuses fois lors des entretiens, les personnes expriment une vision très négative de l'avenir sans les lacs : "ça va mourir », "il n'y aura plus personne » pensent ces usagers. L'arasement des barrages ne peut selon eux que conduire les usagers à l'abandon des lieux, ce qui signifie aussi l'abandon de l'entretien, et partant, le développement de la friche. Ces usagers en quête d'un lien proche à la nature peinent à envisager cette dernière comme seulement sauvage. Il lui faut aussi des humains qui l'entretiennent pour être fréquentable. L'ensemble des occupants de cabanes et maisons rencontrés s'est dit opposé à la disparition des lacs. Malgré des craintes concernant la valeur du foncier ou de l'immobilier et la modification des pratiques dans la vallée, ce sont majoritairement des arguments à l'encontre du bien-fondé même du projet d'effacement qui sont mis en avant. Tous évoquent une décision inutile, coûteuse et risquée. Ces réactions s'inscrivent dans un contexte local conflictuel dans lequel la décision est "présentée comme imposée d'en haut et la méthode mise en place par l'État sévèrement critiquée » (Germaine et Lespez, 2014). L'appropriation forte des lieux est loin d'impliquer un investissement des cabanoniers dans la défense des lacs. Malgré leur opposition, rares sont les occupants à s'impliquer dans l'association des « Amis du Barrage » qui conteste le projet : selon les membres actifs de l'association, sur une centaine d'adhérents seulement une dizaine sont des propriétaires de cabanons. Rares sont ceux à avoir participé à une réunion sur le sujet. Ils se justifient du fait de leur lieu de résidence trop éloigné pour venir participer aux réunions qui ont lieu souvent en semaine, de leur emploi du temps trop chargé ou de la crainte de «ne pas pouvoir se contenir». Ils mentionnent aussi le caractère vain de s'opposer à des décisions actées par l'État et sur lesquelles on ne reviendra pas. Du fait des incertitudes sur la réalisation et les échéances du projet, les positions des cabanoniers ne sont pas tranchées. Elles fluctuent entre trois positions selon le degré d'hostilité au projet d'arasement des barrages et l'attachement aux cabanons.

- Ceux qui ont les constructions les plus élaborées (chalets équipés, maisons) envisagent de continuer à venir dans la vallée et utiliser le logement dans cette nouvelle configuration. Ainsi, ce propriétaire explique : "Ici moi c'est différent parce que je vous ai dit, j'ai l'eau et l'électricité donc j'y viens comme maison de campagne aussi, comme maison de repos. Mais làbas c'est vraiment le bungalow de pêche, la maison qui sert pour la pêche, on vient pour pêcher là-bas, c'est ce qu'on faisait, je vous disais avec mon grand-père: on venait pour pêcher. D'ailleurs quand les pontons étaient abîmés, eh bien c'était le ponton qui était le premier à être réparé. » Plusieurs propriétaires relaient l'idée d'une baisse de valeur immobilière des constructions : "Les maisons, les gens qui vendent les maisons, ils ne vendront pas pareil quoi. Déjà, ça a commencé, le notaire il nous avait expliqué que voilà il y avait plein plein de maisons à vendre du coup parce que depuis l'annonce de la destruction des barrages les gens... »

- D’autres propriétaires disent désirer conserver leur cabane quelque temps au moins " pour voir » par curiosité vis-à-vis du devenir de la vallée. Il s'agit de personnes très attachées à leur cabane pour lesquelles il n'est pas facile d'envisager de l'abandonner dans l'immédiat, bien que la plupart imagine, à moyen terme, ne plus y venir. Il s'agit aussi de personnes attachées à la région pour des raisons souvent familiales, leur cabanon assure une fonction de villégiature qui dépasse la seule activité pêche.

- Enfin, ceux qui possèdent les cabanes les plus modestes et qui l'utilisent principalement pour la pêche ont donné un avis plus tranché : pour eux la cabane n'a plus d'intérêt sans le lac, ils n'envisagent pas de revenir pêcher dans la vallée et évoquent plus 
facilement la destruction ou plus souvent l'abandon. La vidange des lacs provoquera une baisse d'attractivité de la vallée comme l'exprime ce propriétaire pêcheur : "On ne pourra rien faire en attendant, et donc les gens ils vont finir par habiter ailleurs et pour moi ça va mourir ». L'éloignement de l'eau est aussi mis en avant : "Pour nous c'est la fin de nos loisirs, de tout hein. S'il n'y a plus de lac bon... on ne pourra pas se permettre de descendre à la rivière avec la dénivellation qu'il y a. Là, en face il y a 20-15 mètres de profond."

La position des occupants est aussi fonction des sommes engagées dans la construction. Les résidents principaux ne prévoient pas de déménager et les propriétaires ayant investi de l'argent expliquent qu'ils continueront à venir en villégiature si l'état de la vallée le permet. À l'initiative de cinq propriétaires voisins proche du Pont des Biards, trois couples de britanniques possédant chacun une maison et deux propriétaires de cabanes, a adressé un courrier à la préfecture de la Manche en septembre 2014 pour réclamer une indemnisation pour la perte de valeur de leurs propriétés en vue de la disparition des lacs. Un de ces riverains porte le projet de créer un collectif de propriétaires riverains afin d'engager un recours contentieux pour obtenir une indemnisation de l'État. Il légitime cette action par le fait que l'achat des maisons était motivé par la présence du lac: "Toutes ces propriétés-là il ne faut pas rêver, elles ont été achetées... moi le premier je l'ai acheté que pour la pêche, hein, c'est évident. Donc après, j'en fais quoi moi avec une vallée qui va être devant...» La faible interconnaissance des propriétaires au-delà des relations de voisinage immédiat rend cependant difficile la création de ce collectif. Par ailleurs, pour d'autres propriétaires, le faible investissement octroyé à certains abris n'incite pas à y revenir ou récupérer quoi que ce soit. De nombreux occupants relatent aussi ne pas avoir effectué de gros investissements ou travaux depuis l'annonce de l'effacement se désolant alors de voir en quelques années certains éléments (de leur cabane ou des voisines) se délabrer. Certains craignent que ces cabanes ne soient abandonnées, vandalisées et qu'elles ne se transforment en décharge à ciel ouvert, oubliées de ceux qui en furent les fervents occupants.

\section{Conclusion}

Comme l'ont observé d'autres auteurs sur les littoraux, les cabanons constituent un témoignage dans le paysage d'un fort attachement aux lieux. À travers leur construction puis leur utilisation, les occupants ont développé un rapport privilégié et intime au paysage et à la nature des lacs. Cette appropriation est forte mais s'ancre avant tout sur une expérience individuelle et un rapport familial aux lieux. Au-delà des arrangements de voisinage, il n'existe pas de fonctionnement social singulier en dehors d'un mode d'habiter commun dans lequel la vie a le potentiel d'évasion offert par le cabanon et la proximité de la nature est primordiale. Cet attachement conduit d'ailleurs à une forme de privatisation de l'espace excluant d'autres usagers de la vallée. Le cabanon constitue ainsi une forme sociale d'appropriation des espaces de loisirs (Sirost et Raveneau, 2011). L'important investissement fourni par les usagers dans l'entretien des cabanons contraste avec leur faible participation à la définition d'un nouveau projet pour cette portion de la vallée. Ils semblent dessaisis vis-à-vis du devenir de cet espace qui se décide au sein des seules sphères institutionnelles. Indépendamment du cas des propriétaires de cabanons, l'appropriation de la nouvelle vallée s'impose comme un enjeu crucial qui déterminera la réussite du nouveau projet. L'ambition du projet est aussi sociale et politique : il s'agit d' «instaurer (ou restaurer) les sens possibles des paysages » en créant un cadre de vie pour un mieux-être collectif et individuel et non un décor figé (Donadieu et Périgord, 2005). Il s'agira alors d'évaluer si les riverains se reconnaissent dans les nouveaux paysages créés par le projet écologique mais aussi d'identifier les bases matérielles sur lesquelles se construira la réappropriation des lieux : s'appuiera-t-elle sur la mise en place d'une fréquentation de l'espace (cheminements), sur la création de nouveaux habitats (pour les pêcheurs de saumons par exemple)? Cela renvoie également aux bénéficiaires de l'opération qui pourront être les habitants et usagers actuels ou bien des populations extérieures plus lointaines. Au final, c'est la garantie de la redéfinition d'un lien social entre le lieu et de nouveaux usagers qui est donc posée. Quel rôle les cabanons et leurs usagers joueront-ils dans ce processus? L'appropriation actuelle des lieux n'est pas garante d'un investissement dans le projet de vallée. 
Les cabanons sont-ils voués à la disparition une fois les lacs eux-mêmes disparus ou à d'autres formes d'usages et de liens au lieu? Seront-ils amenés à partager l'espace avec d'autres types d'usages?

\section{Remerciements}

Les auteurs souhaitent remercier l'ensemble des personnes des lacs et de la vallée de la Sélune qui ont accepté de répondre à leurs questions. Cet article a été réalisé dans le cadre du programme «Restauration écologique de la rivière Sélune. Paysage, usages, représentations » financé par l'Agence de l'Eau Seine-Normandie (AESN) et piloté par M.-A. Germaine (Univ. Paris Ouest Nanterre La Défense). Il s'agit d'un des quatre volets du programme de suivi scientifique de l'arasement des barrages de la Sélune piloté par J.-L Baglinière (INRA).

\section{Bibliographie}

BaRthéLÉmy C., 2013. La pêche amateur au fil du Rhône et de l'histoire - Usages, savoirs et gestions de la nature, Paris, L'Harmattan, coll. "Eau des villes/Eau des champs », 180 p.

Berque A., 1994. paysage, milieu, histoire, in Berque A. (dir.), Cinq propositions pour une théorie du paysage, Paris, Champ Vallon, p. 13-29.

Brun B., Dufour A.-H., Picon B., Ribéreau-Gayon M.-D., 2001. Cabanes, cabanons et campements. Formes sociales et rapports à la nature en habitat temporaire, Grasse, éd. de Bergier, coll. «Travaux de la Société d'écologie humaine », $336 \mathrm{p}$.

Brunet P., 2001. Inventaire des paysages de Basse-Normandie, Caen, Conseil Régional de Basse-Normandie, DIREN Basse-Normandie, Caen, 2 Tomes, 871 p.

Cabedoce B., Pierson P., 1996, Cent ans d'histoire des jardins ouvriers, 1896-1996, Paris, Créaphis, 221 p.

Cadoret A., 2006. Conflits d'usage liés à l'environnement et réseaux sociaux : enjeux d'une gestion intégrée? Le cas du littoral du Languedoc-Roussillon, Thèse de géographie-aménagement, Université Paul-Valéry, Montpellier III, 586 p.

Claeys-Mekdade C., 2001. Le campeur, le scientifique et le préfet, in Brun B., Dufour A.H., Picon B., RibereauGayon D. (dir.), Cabanes, cabanons et campements. Formes sociales et rapport à la nature en habitat temporaire, Grasse, éd. du Bergier, p. 89-105.

Claeys-Mekdade C., Nicolas L., 1999. De la plage appropriée à la plage patrimonialisée, analyse de pratiques balnéaires « déviantes » sur le littoral camarguais : l'exemple de Piémanson et Beauduc, Méditerranée, Tome 93, 4, p. 27-34.
Corbin A., 2009. L'avènement des loisirs 1850-1960, Flammarion, $626 \mathrm{p}$.

Debarbieux B., 1996. Le lieu, fragment et symbole du territoire, Espaces et Sociétés, no 82-83, p. 13-35.

Donadieu P., Périgord M., 2005. Clés pour le paysage, Ophrys, $367 \mathrm{p}$.

ETHEIS, 2012. Schéma de Développement Durable de la Vallée de la Sélune/Diagnostic et Plan d'Actions, pour la Direction Départementale des Territoires et de la Mer de la Manche, [http://www.manche.gouv.fr].

Féménias D., Evrard B., Sirost O., 2011. Les loisirs sportifs dans l'estuaire de la Seine : natures périurbaines et cadre de vie, Loisir et Société, 34 : 1, p. 67-98.

Frémont A., 1976, La région, espace vécu, Paris, PUF, 223 p.

Geindre F., Helias A., Julien B., Perico B., 2009. Résorption du phénomène de la cabanisation à Vias - Mission d'appui au préfet de la région Languedoc-Roussillon/Ministère de l'Écologie, de l'énergie, du développement durable et de l'aménagement du territoire, $78 \mathrm{p}$.

Germaine M.-A., Lespez L., 2014. Le démantèlement des barrages de la Sélune (Manche). Des réseaux d'acteurs au projet de territoire?, Développement durable et territoires, vol. 5, n³ , décembre 2014, [http://developpementdurable. revues.org/10525].

Germaine M.-A., 2009. Évaluer la diversité des paysages de vallées. Réalisation d'une typologie en Basse-Normandie, Norois, vol. 207, p. 7-19.

Lazzarotтi Olivier, 2006. Habiter : aperçus d'une science géographique, Cahiers de Géographie du Québec, vol. 50, no 139, p. 85-102.

Loubes J.-P., 2001. La cabane, figure géopoétique de l'architecture, in Brun B., Dufour A.H., Picon B., RibereauGayon D. (dir.), Cabanes, cabanons et campements. Formes sociales et rapport à la nature en habitat temporaire, Grasse, éditions du Bergier, p. 89-105.

Maugin A., Perault S., 201 1. Les cabanons du bord de l'Oise, in Raveneau G., Sirost O. (dir.), Anthropologie des abris de loisirs, p. 87-97.

Minvielle P., 2005. La gestion d'un grand site camarguais : les cabanes de Beauduc, Méditerranée, 105, p. 7-17.

Nicolas L., 2008. Beauduc, l'utopie des gratte-plage. Ethnographie d'une communauté de cabaniers sur le littoral camarguais, Marseille, Images en Manœuvres Éditions, 441 p.

Ortar N., 2001. Une autre forme de résidence secondaire : le «chalet », in Brun B., Dufour A.H., Picon B., RibereauGayon D. (dir.), Cabanes, cabanons et campements. Formes sociales et rapport à la nature en habitat temporaire, Grasse, éditions du Bergier, p. 285-290.

Parc naturel régional Oise - Pays de France, 2008. La cabanisation : prévenir, résorber, régulariser, $34 \mathrm{p}$.

Perrin T. (coord.), Cholley F., Vindimian E., 2015. Expertise du projet d'effacement des ouvrages de Vezins et la roche-quiBoit sur la Sélune, CGEDD, CGE, 200 p.

Perrot M., La Soudière (de) M., 1998. La résidence secondaire : un nouveau mode d'habiter la campagne?, Ruralia, vol. 2. 
Picon B., 2001. Les cabanes de l'entre-deux mondes, in Brun B., Dufour A.H., Picon B., Ribereau-Gayon D. (dir.), Cabanes, cabanons et campements. Formes sociales et rapport à la nature en habitat temporaire, Grasse, éditions du Bergier, p. 327-335.

Raveneau G., Sirost O., 201 1, De l'émergence des abris de loisirs aux formes contemporaines, in Raveneau G., Sirost O. (dir.), Anthropologie des abris de loisirs, Presses Paris Ouest, p. $13-28$

Richez J.-C., Strauss L., 1995, Un temps nouveau pour les ouvriers : les congés payés (1930-1960), in Corbin A., L'avènement des loisirs 1850-1960, Paris, Aubier p. 373-411.

Ripoll. F, Veschambre V, 2005, L'appropriation de l'espace comme problématique, Norois, n 195, p. 7-15.

Sirost O., Raveneau G., 201 1, L'abri de loisirs : un territoire de recherches, in Raveneau G., Sirost O. (dir.), Anthropologie des abris de loisirs, Presses Paris Ouest, p. 309-320.
STOck M., 2006, L'hypothèse de l'habiter poly-topique : pratiquer les lieux géographiques dans les sociétés à individus mobiles, EspacesTemps.net, Travaux, [http://www.espacestemps.net/articles/hypothese-habiter-polytopique/].

Sтоск M., 2012, «Faire avec de l'espace » : pour une approche de l'habiter par les pratiques, in Frelat-Kahn B., Lazzaвотті O. (dir.), Habiter, Vers un nowveau concept?, p. 57-76.

Terragone Sevaux et associés, 2011 , État récapitulatif des occupations du domaine concédé, Inventaire réalisé pour EDF, 44 p.

TuAn Y., (1974) 2013, Topophilia : a study of environmental perception, attitudes and values, Columbia, Columbia University Press, $260 \mathrm{p}$. 


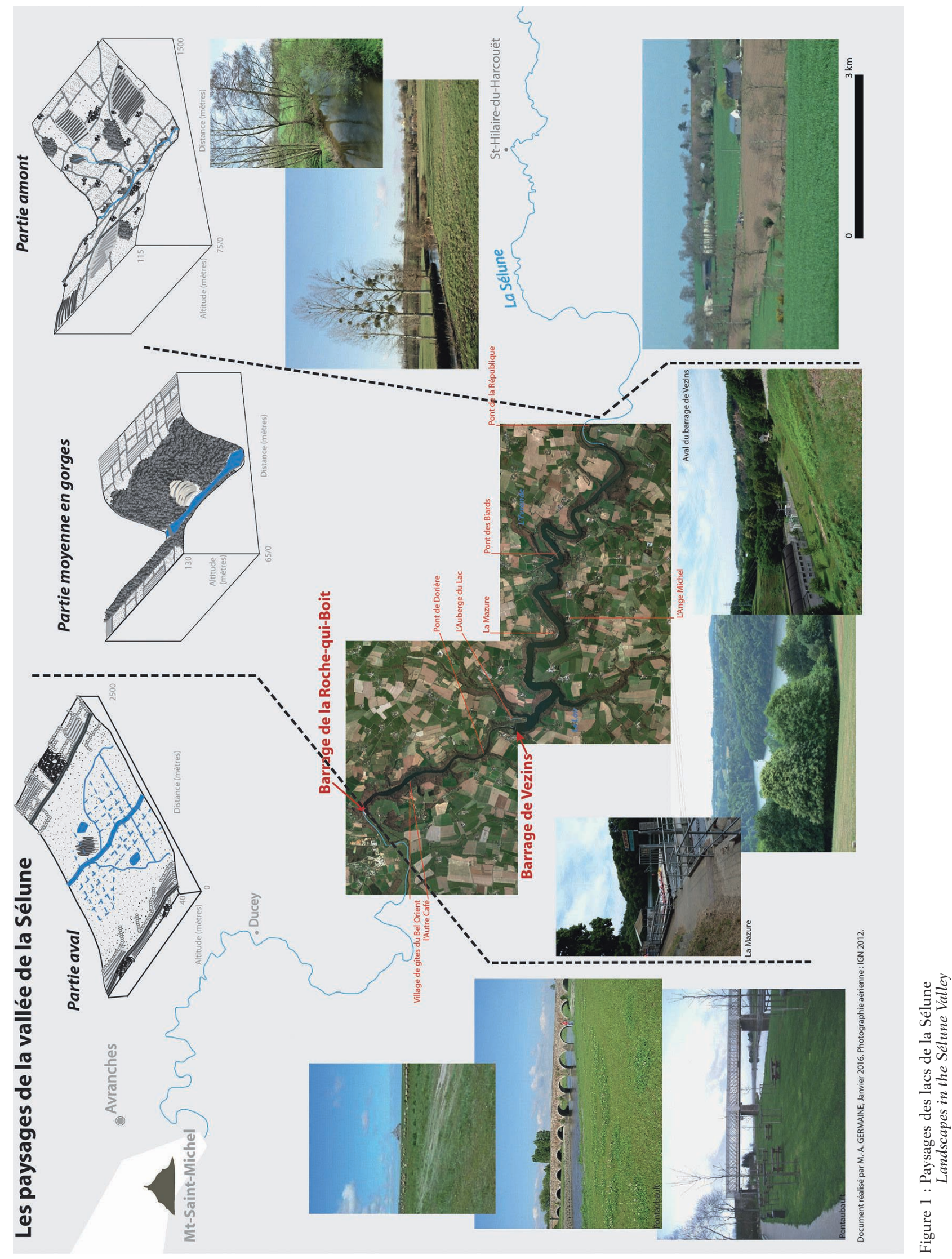




\section{Critères mobilisés pour construire une typologie des constructions principales inventoriées le long des berges des lacs de la Sélune}

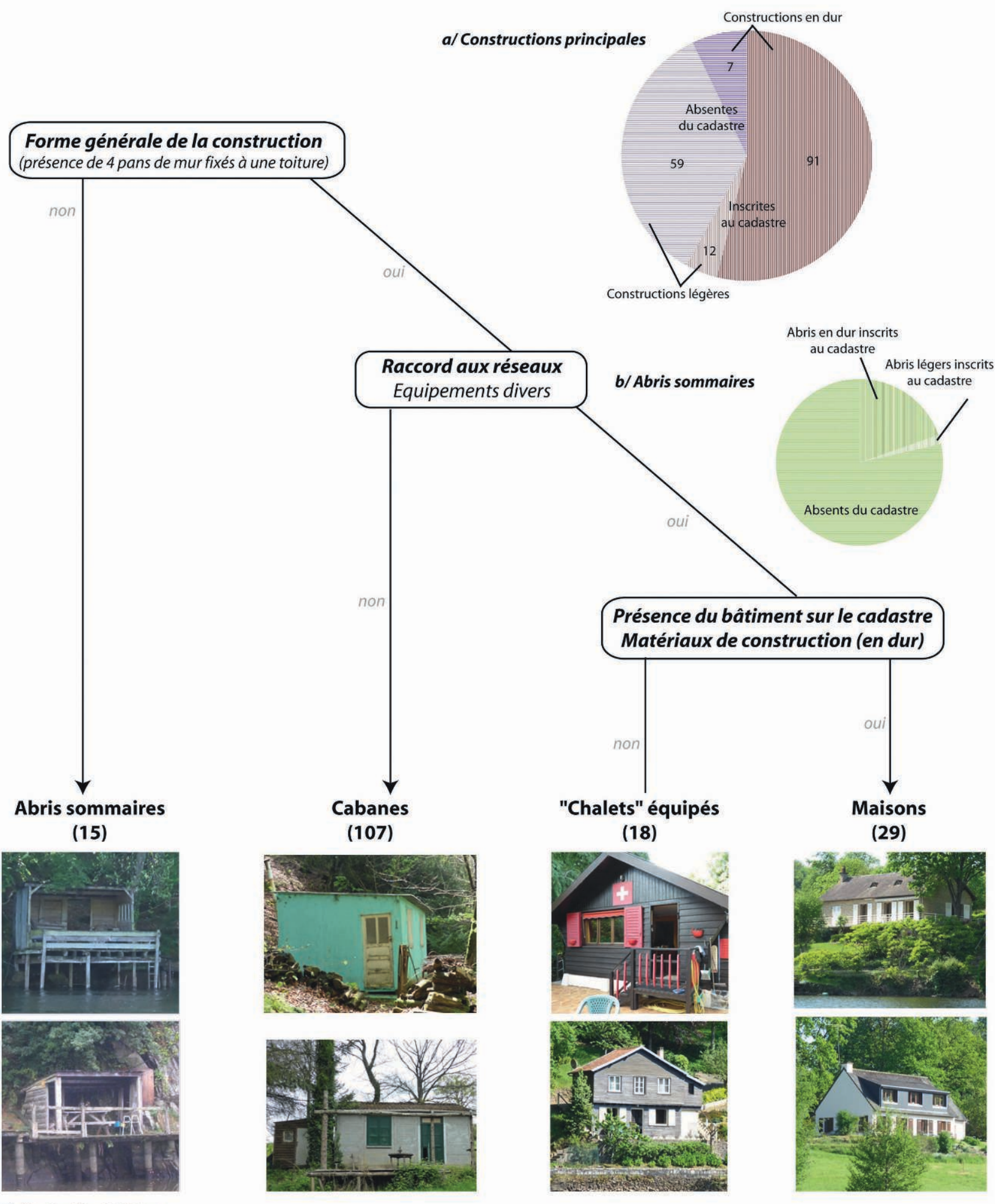

Clichés: Matthieu VIRY, 2014

Figure 2 : Schéma de la construction de la typologie Identification of different hut types 


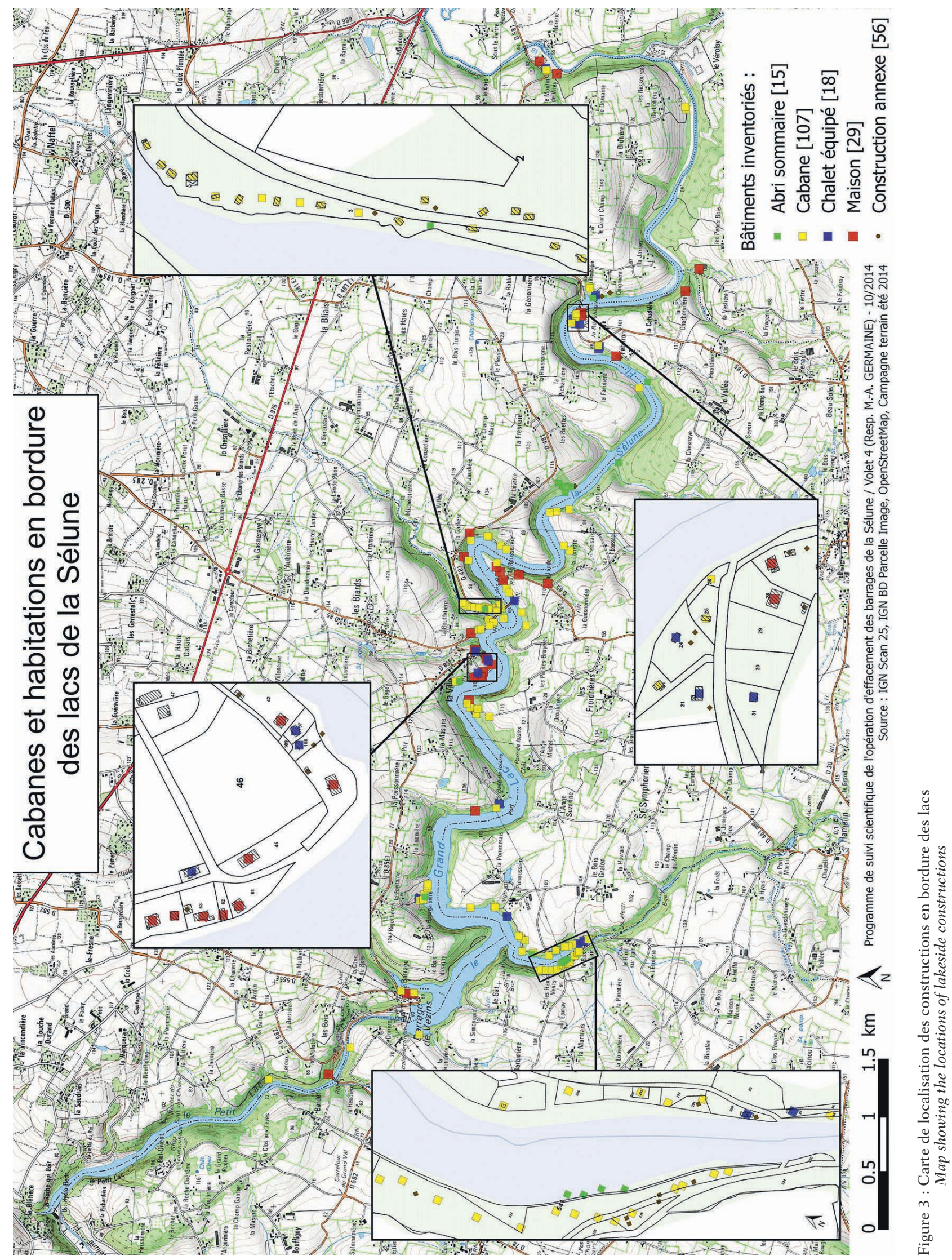


Planche IX (Marie-Anne Germaine, Matthieu VIRY, Marie-Jo MenOZZI - Construction des lieux et rapports à la nature.

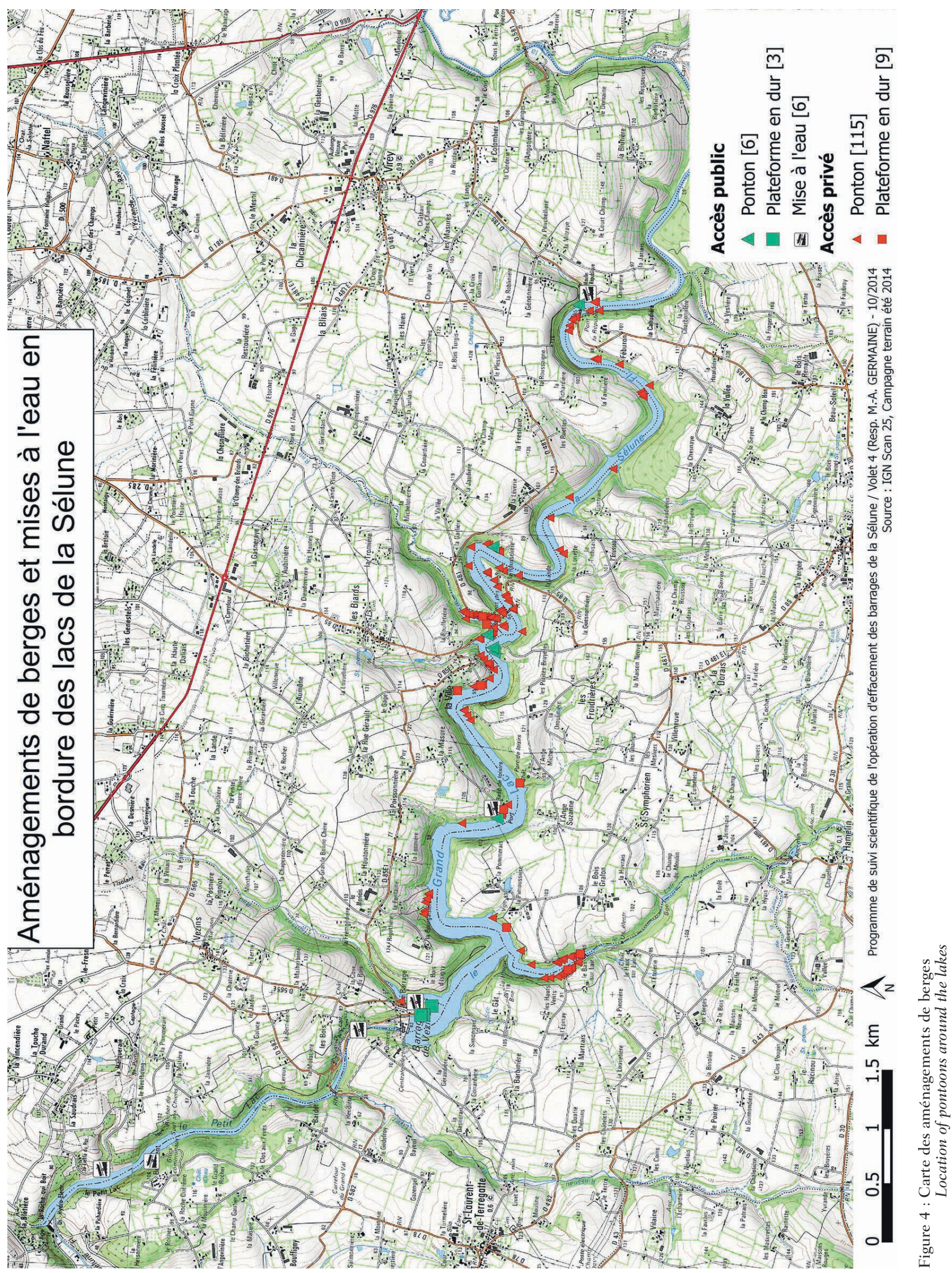

